\title{
The Resolvent of Closed Extensions of Cone Differential Operators
}

\author{
E. Schrohe and J. Seiler
}

\begin{abstract}
We study closed extensions $\underline{A}$ of an elliptic differential operator $A$ on a manifold with conical singularities, acting as an unbounded operator on a weighted $L_{p}$-space. Under suitable conditions we show that the resolvent $(\lambda-\underline{A})^{-1}$ exists in a sector of the complex plane and decays like $1 /|\lambda|$ as $|\lambda| \rightarrow \infty$. Moreover, we determine the structure of the resolvent with enough precision to guarantee existence and boundedness of imaginary powers of $\underline{A}$.

As an application we treat the Laplace-Beltrami operator for a metric with straight conical degeneracy and describe domains yielding maximal regularity for the Cauchy problem $\dot{u}-\Delta u=$ $f, u(0)=0$.
\end{abstract}

\section{Contents}

\begin{tabular}{lll}
\hline 1. Introduction & 1
\end{tabular}

2. Cone differential operators and their closed extensions 3

\begin{tabular}{lll}
\hline 3. & Structure of the resolvent & 10
\end{tabular}

\begin{tabular}{lll}
\hline 4. Spectral invariance and bounded imaginary powers & 16
\end{tabular}

\begin{tabular}{lll}
\hline 5. & The Laplace-Beltrami operator & 17
\end{tabular}

\begin{tabular}{|lll}
\hline $6 . \quad$ Appendix: Parameter-dependent cone pseudodifferential operators & 21
\end{tabular}

References 28

\section{Introduction}

Understanding the resolvent of elliptic differential operators is of central interest for many questions in partial differential equations. Following the approach suggested by Seeley, it is crucial for the analysis of the heat operator or of complex powers. In his classical paper [23], he showed how the parametrix to an elliptic operator on a closed manifold can be constructed as a parameterdependent pseudodifferential operator and how the structure of the parametrix determines the essential properties of the complex powers. He subsequently extended his methods to cover also boundary value problems [24] and proved the boundedness of the purely imaginary powers 225. His results have attracted new interest in connection with modern methods in nonlinear evolution equations, where one requires maximal regularity for the generator of the associated semigroup, which in turn is implied by the boundedness of its purely imaginary powers.

1991 Mathematics Subject Classification. 35J70, 47A10, 58J40.

Key words and phrases. Manifolds with conical singularities, resolvent, maximal regularity. 
In the present paper we study an elliptic differential operator $A$ on a manifold $B$ with conical singularities (a 'cone differential operator'). The investigation of these operators started with the work of Cheeger [2]. Important contributions to the index theory were made in particular by Brüning \& Seeley [1] and Lesch [12]; associated pseudodifferential calculi were devised by Melrose [15], Plamenevskij [17], and Schulze [22].

While the picture of the conical singularity helps the intuition, one prefers to perform the actual analysis on a manifold $\mathbb{B}$ with boundary, thought of as the blow-up of $B$. A cone differential operator of order $\mu$ by definition is an operator that can be written in the form

$$
A=t^{-\mu} \sum_{j=0}^{\mu} a_{j}(t)\left(-t \partial_{t}\right)^{j}
$$

in a neighborhood of the boundary. Here $t$ is a boundary defining function and $a_{j}$ a smooth family of differential operators of order $\mu-j$ on $\partial \mathbb{B}$.

We consider $A$ as an unbounded operator acting in a (weighted) $L_{p}$-space. Our goal is to find conditions which ensure the existence of the resolvent $(A-\lambda)^{-1}$ in a sector of the complex plane with decay like $1 /|\lambda|$ as $|\lambda| \rightarrow \infty$ and to determine its structure with enough precision to construct complex powers and to show their boundedness for purely imginary exponents. We work with a variant of Schulze's cone calculus because the concept of meromorphic Mellin symbols makes it easy to describe the connection between operators and function spaces with asymptotics.

A cone differential operator in general has many closed extensions, see e.g. Lesch [12, Section 1.3]. While, a priori, there is no preference for any of these from the analytical point of view, it is obvious that only for few of them the resolvent will have good properties. One basic problem therefore is to determine all possible choices. Our Theorem 2.8 completes Lesch's results in that we obtain an explicit formula for the domain of the maximal extension in the general situation.

Extending Theorem 3.14 from [20], we next clarify the structure of the inverse of a bijective closed extension of $A$. In Theorem 3.4 we show how $A^{-1}$ can be decomposed as the sum of two operators in usual cone calculi for different weight data.

We then turn to the analysis of the resolvent. In order to keep the exposition short and the proofs transparent we restrict ourselves to the case where the coefficients $a_{j}$ of the operator $A$ are constant for small $t$. The general case will be treated in a subsequent publication.

Following a standard technique, we replace the spectral parameter $\lambda$ by $\eta^{\mu}$, where $\mu$ is the order of $A$, and $\eta$ varies in a corresponding sector of $\mathbb{C}$. In close analogy to Theorem 3.4 we prove in Theorem 3.5 that $\left(A-\eta^{\mu}\right)^{-1}$ is the sum of two parameter-dependent cone operators; the parameter space is the new $\eta$-sector. In order to establish this fact we have to make assumptions which are restrictive but nevertheless seem natural in this context: Clearly, we have to ask for the invertibility of the principal pseudodifferential symbol of $A-\eta^{\mu}$ in the sector, with a certain uniformity as one approaches the singularity. Moreover, we require the invertibility of $\widehat{A}-\eta^{\mu}$, where $\widehat{A}$ is the 'model cone operator' associated to $A$. It is given by $\widehat{A}=t^{-\mu} \sum_{j=0}^{\mu} a_{j}(0)\left(-t \partial_{t}\right)^{j}$ on $\mathbb{R}_{+} \times \partial \mathbb{B}$ and reflects the behavior of $A$ near the singular point; $\widehat{A}$ acts on a domain linked to that of $A$. As $\widehat{A}-\eta^{\mu}$ can be considered the analog of an edge principal symbol for $A-\eta^{\mu}$, its invertibility appears to be necessary for the above result. Finally, we assume for technical reasons that the domain of $A$ (or more precisely the associated domain of $\widehat{A}$ ) is invariant under dilations ('saturated' in the language of Gil and Mendoza [10]).

It follows from Theorem 5.1 and Remark 5.5 in [3] that the structure of the resolvent we obtain from Theorem 3.5 is precisely that required for the construction of complex powers and implies the boundedness of the purely imaginary powers; we can hence extend the results of that paper as well as those in [4 to this new class of operators. 
The idea of analyzing the resolvent of a cone differential operator in terms of a suitable pseudodifferential calculus is not new. In fact, writing the resolvent as a parameter-dependent cone operator can be seen as a special case of the edge parametrix construction, see Schulze [6], Section 9.3.3, Theorem 6]. Moreover, Gil [7], [8], and Loya [13], [14], also in joint work [9], used this technique to derive results on heat invariants, complex powers, and noncommutative residues. While these are important theorems, there is one draw-back: In all articles, the authors rely on a special form of the above ellipticity condition, namely the invertibility of $\widehat{A}-\eta^{\mu}$, acting between weighted Mellin Sobolev spaces. One can show, however, that this assumption fails in many cases, e.g. for the Laplace-Beltrami operator in dimensions $\leq 4$, acting in $L^{2}$ with respect to any metric that has a straight conical singularity. Roughly speaking, this approach works only for the minimal (and hence by duality for the maximal) extension. The new point here is that we can now treat all closed extensions with dilation invariant (saturated) domains, opening the way for the analysis of larger classes of operators.

As an application we study the Laplacian in weighted $L_{p}$-spaces, $1<p<\infty$. Combining our analysis with techniques of Gil and Mendoza [10, we show in Theorems 5.6 and 5.7 how one can always choose the domain in such a way that the above ellipticity conditions are fulfilled. This yields maximal regularity for the Cauchy problem $\dot{u}-\Delta u=f$ on $] 0, T[, u(0)=0$, which is the starting point for many results in nonlinear evolution equations.

\section{Cone differential operators and their closed extensions}

2.1. Operators on $\mathbb{B}$. Let $\mathbb{B}$ be a smooth, compact manifold with boundary. A $\mu$-th order differential operator $A$ with smooth coefficients acting on sections of a vector bundle $E$ over the interior of $\mathbb{B}$ is called a cone differential operator if, near the boundary, it has the form

$$
A=t^{-\mu} \sum_{j=0}^{\mu} a_{j}(t)\left(-t \partial_{t}\right)^{j}, \quad a_{j} \in \mathcal{C}^{\infty}\left(\left[0,1\left[, \operatorname{Diff}^{\mu-j}(\partial \mathbb{B})\right) .\right.\right.
$$

In more detail: We assume (as we may) that $E$ respects the product structure near the boundary, i.e. $E$ is the pull-back of a vector bundle $E_{\partial}$ over $\partial \mathbb{B}$ under the canonical projection $[0,1[\times \partial \mathbb{B} \rightarrow \partial \mathbb{B}$. The coefficients $a_{j}(t)$ then are differential operators acting on sections of $E_{\partial}$. In order to keep the exposition simple, however, we shall not indicate the bundles in the notation.

Besides the standard pseudodifferential principal symbol $\sigma_{\psi}^{\mu}(A) \in \mathcal{C}^{\infty}\left(T^{*}\right.$ int $\left.\mathbb{B} \backslash 0\right)$, we associate with $A$ two other symbols: First, there is the rescaled symbol $\widetilde{\sigma}_{\psi}^{\mu}(A) \in \mathcal{C}^{\infty}\left(\left(T^{*} \partial \mathbb{B} \times \mathbb{R}\right) \backslash 0\right)$ which, in local coordinates, is given by

$$
\widetilde{\sigma}_{\psi}^{\mu}(A)(x, \xi, \tau)=\sum_{j=0}^{\mu} \sigma_{\psi}^{\mu-j}\left(a_{j}\right)(0, x, \xi)(-i \tau)^{j} .
$$

Secondly, we have the conormal symbol $\sigma_{M}^{\mu}(A)$ defined by

$$
\sigma_{M}^{\mu}(A)(z)=\sum_{j=0}^{\mu} a_{j}(0) z^{j}, \quad z \in \mathbb{C} .
$$

It is a polynomial in $z$ of degree at most $\mu$ with values in differential operators on $\partial \mathbb{B}$ of order at most $\mu$. In particular, $\sigma_{M}^{\mu}(A) \in \mathcal{A}\left(\mathbb{C}, \mathcal{L}\left(H_{p}^{s}(\partial \mathbb{B}), \mathcal{L}\left(H_{p}^{s-\mu}(\partial \mathbb{B})\right)\right)\right.$ for all $s \in \mathbb{R}, 1<p<\infty$, where $\mathcal{A}(\mathbb{C}, X)$ denotes the holomorphic, $X$-valued functions on $\mathbb{C}$.

Let us introduce some notions we shall frequently use throughout this paper.

A cut-off function, generally denoted by $\omega, \omega_{0}, \omega_{1}$, or $\sigma, \sigma_{0}, \sigma_{1}$, is a non-negative decreasing function in $\mathcal{C}_{\text {comp }}^{\infty}([0,1[)$, which is identically 1 near zero.

DEFINITION 2.1. $\quad$ a) $A$ is called $\mathbb{B}$-elliptic if both $\sigma_{\psi}^{\mu}(A)$ and $\widetilde{\sigma}_{\psi}^{\mu}(A)$ are pointwise invertible.

b) $A$ is said to have $t$-independent coefficients near the boundary if the functions $a_{j}$ in (2.1) are constant in $t$. 
The operator $A$ induces continuous actions

$$
A: \mathcal{H}_{p}^{s, \gamma}(\mathbb{B}) \longrightarrow \mathcal{H}_{p}^{s-\mu, \gamma-\mu}(\mathbb{B}), \quad s, \gamma \in \mathbb{R}, 1<p<\infty,
$$

in a scale of Sobolev spaces which is defined as follows:

Definition 2.2. Let $s \in \mathbb{N}_{0}$. The space of all distributions $u \in H_{p, l o c}^{s}(\operatorname{int} \mathbb{B})$ with

$$
t^{\frac{n+1}{2}-\gamma}\left(t \partial_{t}\right)^{k} \partial_{x}^{\alpha}(\omega u)(t, x) \in L_{p}\left(\left[0,1\left[\times \partial \mathbb{B}, \frac{d t}{t} d x\right) \quad \forall k+|\alpha| \leq s\right.\right.
$$

is denoted by $\mathcal{H}_{p}^{s, \gamma}(\mathbb{B})$. Here, $\omega$ denotes an arbitrary cut-off function.

This definition extends to real $s$, yielding a scale of Banach spaces (Hilbert spaces in case $p=2$ ) with two properties we want to mention explicitly: The embedding $\mathcal{H}_{p}^{s^{\prime}, \gamma^{\prime}}(\mathbb{B}) \hookrightarrow \mathcal{H}_{p}^{s, \gamma}(\mathbb{B})$ is continuous for $s^{\prime} \geq s, \gamma^{\prime} \geq \gamma$, and compact if $s^{\prime}>s, \gamma^{\prime}>\gamma$; the scalar-product of $\mathcal{H}_{2}^{0,0}(\mathbb{B})$ induces an identification of the dual space $\left(\mathcal{H}_{p}^{s, \gamma}(\mathbb{B})\right)^{\prime}$ with $\mathcal{H}_{p^{\prime}}^{-s,-\gamma}(\mathbb{B})$, where $p^{\prime}$ is the dual number to $p$, i.e. $\frac{1}{p}+\frac{1}{p^{\prime}}=1$

Instead of considering $A$ as a continuous operator in the Sobolev spaces, we shall now study the closed extensions of the unbounded operator

$$
A: \mathcal{C}_{\mathrm{comp}}^{\infty}(\operatorname{int} \mathbb{B}) \subset \mathcal{H}_{p}^{0, \gamma}(\mathbb{B}) \longrightarrow \mathcal{H}_{p}^{0, \gamma}(\mathbb{B})
$$

In the sequel $A$ will be assumed to be $\mathbb{B}$-elliptic and of positive order $\mu>0$. In the upcoming Sections 2.2, 2.3 we shall give an explicit description of all possible closed extensions of $A$. We shall need a few basic facts about the cone calculus which may be found in the short introduction 27. We refer in particular to 27, Section 2.4, for the notion of Mellin pseudodifferential operators $\mathrm{op}_{M}^{\delta}$ and their mapping properties on the spaces $\mathcal{H}_{p}^{s, \gamma}(\mathbb{B})$. Some material can also be found in the appendix; these two points, for example are covered by (6.13) and Remark 6.16. We let $\omega$ denote an arbitratry cut-off function.

2.2. The minimal extension. The following result was shown in [10], Proposition 3.6. We give here a short proof, using some results of [12].

Proposition 2.3. The domain of the closure $A_{\min }=A_{\min }^{\gamma, p}$ of $A$ from (2.3) is

$$
\begin{aligned}
\mathcal{D}\left(A_{\min }\right) & =\mathcal{D}\left(A_{\max }\right) \cap \bigcap_{\varepsilon>0} \mathcal{H}_{p}^{\mu, \gamma+\mu-\varepsilon}(\mathbb{B}) \\
& =\left\{u \in \bigcap_{\varepsilon>0} \mathcal{H}_{p}^{\mu, \gamma+\mu-\varepsilon}(\mathbb{B}) \mid t^{-\mu} \sum_{j=0}^{\mu} a_{j}(0)\left(-t \partial_{t}\right)^{j}(\omega u) \in \mathcal{H}_{p}^{0, \gamma}(\mathbb{B})\right\} .
\end{aligned}
$$

In particular,

$$
\mathcal{H}_{p}^{\mu, \gamma+\mu}(\mathbb{B}) \hookrightarrow \mathcal{D}\left(A_{\min }\right) \hookrightarrow \mathcal{H}_{p}^{\mu, \gamma+\mu-\varepsilon}(\mathbb{B}) \quad \forall \varepsilon>0 .
$$

We have $\mathcal{D}\left(A_{\min }\right)=\mathcal{H}_{p}^{\mu, \gamma+\mu}(\mathbb{B})$ if and only if the conormal symbol $\sigma_{M}^{\mu}(A)(z)$ is invertible for all $z$ with $\operatorname{Re} z=\frac{n+1}{2}-\gamma-\mu$.

Proof. According to [12], Proposition 1.3.12, we may assume that $A$ has $t$-independent coefficients near the boundary. Now let $u \in \mathcal{D}\left(A_{\min }\right)$, i.e. there exists a sequence of functions $u_{n} \in \mathcal{C}_{\text {comp }}^{\infty}($ int $\mathbb{B})$ such that $u_{n} \rightarrow u$ and $A u_{n} \rightarrow A u$ with convergence in $\mathcal{H}_{p}^{0, \gamma}(\mathbb{B})$. Choose a cut-off function $\widetilde{\omega}$ with $\omega \widetilde{\omega}=\omega$, and let $B=\widetilde{\omega} \operatorname{op}_{M}^{\gamma+\mu-\varepsilon-\frac{n}{2}}\left(\sigma_{M}^{\mu}(A)^{-1}\right) t^{\mu}$ with arbitrarily small $\varepsilon>0$ (and $\varepsilon=0$ in case of the invertibility of the conormal symbol). It follows from elliptic regularity that $u_{n} \rightarrow u$ in $H_{p, \text { loc }}^{\mu}(\operatorname{int} \mathbb{B})$. Thus $(1-\omega) u_{n} \rightarrow(1-\omega) u$ in $\mathcal{H}_{p}^{\mu, \gamma+\mu}(\mathbb{B})$ and $A\left(\omega u_{n}\right) \rightarrow A(\omega u)$ in $\mathcal{H}_{p}^{0, \gamma}(\mathbb{B})$. Therefore $\omega u$ belongs to $\mathcal{D}\left(A_{\min }\right)$ and

$$
\omega u \stackrel{n \rightarrow \infty}{\stackrel{n}{\longleftarrow}} \omega u_{n}=B A\left(\omega u_{n}\right) \stackrel{n \rightarrow \infty}{\longrightarrow} B A(\omega u)
$$

in $\mathcal{H}_{p}^{0, \gamma}(\mathbb{B})$. The continuity of $B: \mathcal{H}_{p}^{0, \gamma}(\mathbb{B}) \rightarrow \mathcal{H}_{p}^{\mu, \gamma+\mu-\epsilon}(\mathbb{B})$ implies that

$$
\mathcal{D}\left(A_{\min }\right) \subset \mathcal{D}\left(A_{\max }\right) \cap \bigcap_{\varepsilon>0} \mathcal{H}_{p}^{\mu, \gamma+\mu-\varepsilon}(\mathbb{B})=: V
$$


Since $A$ has constant coefficients, $\mathcal{D}\left(A_{\max }\right)=\mathcal{D}\left(A_{\min }\right) \oplus \mathcal{E}$, where $\mathcal{E}$ has zero intersection with $\bigcap_{\varepsilon>0} \mathcal{H}_{p}^{\mu, \gamma+\mu-\varepsilon}(\mathbb{B})$, see $1 \mathbf{1 2}$, Proposition 1.3.11. From this we immediately obtain $\mathcal{D}\left(A_{\min }\right)=V$.

2.3. The maximal extension. Before characterizing the domain of the maximal extension, we shall discuss a certain type of operators, namely those of the form

$$
G=\omega\left(\operatorname{op}_{M}^{\gamma_{1}-\frac{n}{2}}(g)-\operatorname{op}_{M}^{\gamma_{2}-\frac{n}{2}}(g)\right): \mathcal{C}_{\text {comp }}^{\infty}\left(\partial \mathbb{B}^{\wedge}\right) \longrightarrow \mathcal{C}^{\infty}(\operatorname{int} \mathbb{B})
$$

Here, $\partial \mathbb{B}^{\wedge}:=\mathbb{R}_{+} \times \partial \mathbb{B}$. Moreover, $g$ is a meromorphic Mellin symbol with asymptotic type $P$ as in 27] or Section 6.5. Let

$$
\sum_{k=0}^{n_{p}} R_{p k}(z-p)^{-(k+1)}, \quad R_{p k} \in L^{-\infty}(\partial \mathbb{B})
$$

denote the principal part of $g$ around $p \in \pi_{\mathbb{C}} P$. Recall that the $R_{p k}$ have finite rank by definition.

LEMma 2.4. Let $G$ be as in (2.5) with $\gamma_{1}<\gamma_{2}$, and $R_{p k}$ as in (2.6). Then $G$ is of finite rank and, for $u \in \mathcal{C}_{\mathrm{comp}}^{\infty}\left(\partial \mathbb{B}^{\wedge}\right)$,

$$
(G u)(t, x)=\omega(t) \sum_{\substack{p \in \pi_{\mathbb{C} P} \\-\gamma_{2}<\operatorname{Re} p-\frac{n+1}{2}<-\gamma_{1}}} \sum_{l=0}^{n_{p}} \zeta_{p l}(u)(x) t^{-p}(\log t)^{l}
$$

with the linear maps $\zeta_{p l}: \mathcal{C}_{\mathrm{comp}}^{\infty}\left(\partial \mathbb{B}^{\wedge}\right) \rightarrow \operatorname{im} R_{p l}+\ldots+\operatorname{im} R_{p n_{p}} \subset \mathcal{C}^{\infty}(\partial \mathbb{B})$ given by

$$
\zeta_{p l}(u)(x)=\sum_{k=l}^{n_{p}} \frac{(-1)^{l}}{l !(k-l) !} R_{p k} \frac{\partial^{k-l}}{\partial z^{k-l}}(\mathcal{M} u)(p, x)
$$

where $\mathcal{M}=\mathcal{M}_{t \rightarrow z}$ denotes the Mellin transform.

The proof is a straightforward consequence of the residue theorem, since

$$
(G u)(t, x)=\left(\int_{\Gamma_{\frac{n+1}{2}-\gamma_{1}}}-\int_{\Gamma_{\frac{n+1}{2}-\gamma_{2}}}\right) t^{-z} g(z)(\mathcal{M} u)(z, x) d z=\int_{\mathcal{C}} t^{-z} g(z)(\mathcal{M} u)(z, x) d z
$$

with a path $\mathcal{C}$ simply surrounding the poles of $g$ in the strip $\frac{n+1}{2}-\gamma_{2}<\operatorname{Re} z<\frac{n+1}{2}-\gamma_{1}$. For the detailed calculations and an expression for $\operatorname{rank} G$ see $[\mathbf{1 2}$. The residue theorem also implies that we could replace $g$ by $g+h$ for any $h \in M_{O}^{\mu}(\partial \mathbb{B})$ without changing $G$.

REMARK 2.5. Let $\gamma$ with $\gamma_{1}<\gamma<\gamma_{2}$ be given and let $G_{j}=\omega\left(\operatorname{op}_{M}^{\gamma_{j}-\frac{n}{2}}(g)-\operatorname{op}_{M}^{\gamma-\frac{n}{2}}(g)\right)$. Then $G=G_{1}-G_{2}$ and

$$
\operatorname{im} G=\operatorname{im} G_{1} \oplus \operatorname{im} G_{2}
$$

In fact, by the previous lemma, the images on the right-hand side have trivial intersection, and $G_{2} u_{2}$ only depends on finitely many Taylor coefficients of the Mellin transform $\mathcal{M} u_{2}$ in the poles of $g$ lying in the strip $\frac{n+1}{2}-\gamma_{2}<\operatorname{Re} z<\frac{n+1}{2}-\gamma$. The analogous statement holds for $G_{1} u_{1}$. Then the result follows from the following observation: Given finitely many points $p_{1}, \ldots, p_{N} \in \mathbb{C}$ and, in each of these, a finite number of Taylor coefficients, there exists a $u \in \mathcal{C}_{\text {comp }}^{\infty}\left(\mathbb{R}_{+}\right)$such that the Taylor expansion of $\mathcal{M} u$ in each $p_{j}$ starts with these prescribed values.

Now let $A$ be as in (2.1) and set

$$
f_{l}(z)=\frac{1}{l !} \sum_{j=0}^{\mu}\left(d_{t}^{l} a_{j}\right)(0) z^{j}, \quad l=0, \ldots, \mu-1 .
$$

In particular, $f_{0}=\sigma_{M}^{\mu}(A)$ is the conormal symbol of $A$. Due to the $\mathbb{B}$-ellipticity of $A, f_{0}$ is meromorphically invertible and $f_{0}^{-1}$ can be written as the sum of a meromorphic Mellin symbol 
and a holomorphic symbol in $M_{O}^{-\mu}(\partial \mathbb{B}$ ) (see Theorem 6 in Section 2.3.1 of [22]). We now define recursively

$$
g_{0}=f_{0}^{-1}, \quad g_{l}=-\left(T^{-l} f_{0}^{-1}\right) \sum_{j=0}^{l-1}\left(T^{-j} f_{l-j}\right) g_{j}, \quad l=1, \ldots, \mu-1,
$$

with $T^{\sigma}, \sigma \in \mathbb{R}$, acting on meromorphic functions by $\left(T^{\sigma} f\right)(z)=f(z+\sigma)$.

Moreover, choose an $\varepsilon>0$ so small that every pole $p$ of one of the symbols $g_{0}, \ldots, g_{\mu-1}$, either lies on one of the lines $\Gamma_{\frac{n+1}{2}-\gamma-\mu+k}, k=0, \ldots, \mu$, or has a distance to each of these lines which is larger then $\varepsilon$.

Definition 2.6. Let $g_{0}, \ldots, g_{\mu-1}$ be as in (2.8) and $\varepsilon>0$ as described before. Then we set

$$
\mathcal{E}=\mathcal{E}_{A}^{\gamma}=\operatorname{im} G_{0}+\ldots+\operatorname{im} G_{\mu-1},
$$

where the operators $G_{k}=\sum_{l=0}^{k} G_{k l}: \mathcal{C}_{\mathrm{comp}}^{\infty}\left(\partial \mathbb{B}^{\wedge}\right) \rightarrow \mathcal{H}_{p}^{\infty, \gamma+\varepsilon}(\mathbb{B})$ are defined by

$$
G_{0}=G_{00}=\omega\left(\mathrm{op}_{M}^{\gamma+\mu+\varepsilon-1-\frac{n}{2}}\left(g_{0}\right)-\mathrm{op}_{M}^{\gamma+\mu-\varepsilon-\frac{n}{2}}\left(g_{0}\right)\right)
$$

and if $1 \leq k \leq \mu-1,0 \leq l \leq k$,

$$
G_{k l}=\omega t^{l}\left(\mathrm{op}_{M}^{\gamma+\mu+\varepsilon-k-1-\frac{n}{2}}\left(g_{l}\right)-\mathrm{op}_{M}^{\gamma+\mu+\varepsilon-k-\frac{n}{2}}\left(g_{l}\right)\right) .
$$

The space $\mathcal{E}$ is a finite-dimensional subspace of $\mathcal{C}^{\infty, \gamma+\varepsilon}(\mathbb{B})$ and consists of functions of the form

$$
u(t, x)=\omega(t) \sum_{j=0}^{N} \sum_{k=0}^{l_{j}} u_{j k}(x) t^{-q_{j}} \log ^{k} t
$$

with smooth functions $u_{j k} \in \mathcal{C}^{\infty}(\partial \mathbb{B})$ and complex numbers $q_{j}$ with

$$
\frac{n+1}{2}-\gamma-\mu \leq \operatorname{Re} q_{j}<\frac{n+1}{2}-\gamma .
$$

Note that in case $A$ has constant coefficients we have, due to Remark 2.5,

$$
\mathcal{E}=\operatorname{im} G_{00} \oplus \ldots \oplus \operatorname{im} G_{(\mu-1) 0}=\operatorname{im} \omega\left(\mathrm{op}_{M}^{\gamma+\mu-\varepsilon-\frac{n}{2}}\left(\sigma_{M}^{\mu}(A)^{-1}\right)-\mathrm{op}_{M}^{\gamma+\varepsilon-\frac{n}{2}}\left(\sigma_{M}^{\mu}(A)^{-1}\right)\right) ;
$$

in particular, we have twice strict inequality ' $<$ ' in (2.10). For $A$ having non-constant coefficients, equality in (2.10) is possible, see Example 2.9, below.

Proposition 2.7. For any $0 \leq k \leq \mu-1$ let $u_{k 1}, \ldots, u_{k n_{k}} \in \mathcal{C}_{\text {comp }}^{\infty}\left(\partial \mathbb{B}^{\wedge}\right)$ be chosen such that $\left\{G_{k 0} u_{k j} \mid 1 \leq j \leq n_{k}\right\}$ is a basis of $\operatorname{im} G_{k 0}$. Then

$$
\left\{G_{k} u_{k j} \mid 0 \leq k \leq \mu-1,1 \leq j \leq n_{k}\right\} \subset \mathcal{E}
$$

is a set of linearly independent functions such that

$$
\operatorname{span}\left\{G_{k} u_{k j} \mid 0 \leq k \leq \mu-1,1 \leq j \leq n_{k}\right\} \cap \mathcal{D}\left(A_{\min }\right)=\{0\} .
$$

In particular,

$$
\operatorname{dim} \mathcal{E} \geq \operatorname{dimim} \omega\left(\mathrm{op}_{M}^{\gamma+\varepsilon-\frac{n}{2}}\left(\sigma_{M}^{\mu}(A)^{-1}\right)-\mathrm{op}_{M}^{\gamma+\mu-\varepsilon-\frac{n}{2}}\left(\sigma_{M}^{\mu}(A)^{-1}\right)\right) .
$$

We have equality at least in the cases where $A$ has constant coefficients near the boundary or $\sigma_{M}^{\mu}(A)^{-1}$ has no pole on the line $\operatorname{Re} z=\frac{n+1}{2}-\gamma-\mu$.

Proof. Let $\alpha_{j k} \in \mathbb{C}$ and

$$
\sum_{k=0}^{\mu-1} \sum_{j=1}^{n_{k}} \alpha_{j k} G_{k} u_{k j}=u \in \mathcal{D}\left(A_{\min }\right) \subset \mathcal{H}_{p}^{0, \gamma+\mu-\varepsilon}(\mathbb{B}) .
$$


Setting $l=\mu-1$, we obtain

$$
\sum_{j=1}^{n_{l}} \alpha_{l j} G_{l 0} u_{l j}=u-\sum_{k=0}^{l-1} \sum_{j=1}^{n_{k}} \alpha_{j k} G_{k} u_{k j}-\sum_{j=1}^{n_{l}} \alpha_{l k}\left(G_{l}-G_{l 0}\right) u_{l j}
$$

The right-hand side belongs to $\mathcal{H}_{p}^{0, \gamma+\mu-\varepsilon}(\mathbb{B})+\mathcal{H}_{p}^{0, \gamma+1+\varepsilon}(\mathbb{B})$. The intersection of this space with

$\operatorname{im} G_{l 0}$ is trivial, hence $\sum_{j=1}^{n_{l}} \alpha_{l j} G_{l 0} u_{l j}=0$. Therefore $\alpha_{l j}=0$ for all $1 \leq j \leq n_{l}$, since the $G_{l 0} u_{l j}$ are linearly independent by assumption. Iterating this process (i.e. taking $l=\mu-2, l=\mu-3$, etc.), we see that all $\alpha_{j k}$ must equal zero.

If $A$ has constant coefficients, the result on the dimension follows from (2.11); the second identity in (2.11) is always true and yields the lower bound for $\operatorname{dim} \mathcal{E}$. The remaining claim we shall obtain as a by-product of the following theorem.

THEOREM 2.8. The domain of the maximal extension $A_{\max }=A_{\max }^{\gamma, p}$ of $A$ from (2.3) $i s$

$$
\mathcal{D}\left(A_{\text {max }}\right)=\mathcal{D}\left(A_{\text {min }}\right)+\mathcal{E}
$$

with $\mathcal{E}$ from Definition 2.6. Recall that $\mathcal{E}$ does not depend on $1<p<\infty$. The sum is direct at least in the cases where $A$ has constant coefficients near the boundary or $\sigma_{M}^{\mu}(A)^{-1}$ has no pole on the line $\operatorname{Re} z=\frac{n+1}{2}-\gamma-\mu$. In any case,

$$
\mathcal{D}\left(A_{\min }\right) \cap \mathcal{E} \subset \operatorname{im} \omega\left(\operatorname{op}_{M}^{\gamma+\mu-\varepsilon-\frac{n}{2}}\left(\sigma_{M}^{\mu}(A)^{-1}\right)-\operatorname{op}_{M}^{\gamma+\mu+\varepsilon-\frac{n}{2}}\left(\sigma_{M}^{\mu}(A)^{-1}\right)\right) .
$$

Consequently, any closed extension $\underline{A}$ in $\mathcal{H}_{p}^{0, \gamma}(\mathbb{B})$ is given by the action of $A$ on a domain

$$
\mathcal{D}(\underline{A})=\mathcal{D}\left(A_{\min }\right)+\underline{\mathcal{E}}, \quad \underline{\mathcal{E}} \text { subspace of } \mathcal{E} .
$$

ProOF. For a cut-off function $\widetilde{\omega}$ supported sufficiently close to zero, the operator $\widetilde{A}=\widetilde{\omega} t^{-\mu}$ $\sum_{j=0}^{\mu} a_{j}(0)\left(-t \partial_{t}\right)^{j}+(1-\widetilde{\omega}) A$ is still $\mathbb{B}$-elliptic, and $\mathcal{D}\left(\widetilde{A}_{\min }\right)=\mathcal{D}\left(A_{\min }\right)$ by $[\mathbf{1 2}$, Proposition 1.3.12. Moreover,

$$
\mathcal{D}\left(\widetilde{A}_{\text {max }}\right)=\mathcal{D}\left(\widetilde{A}_{\text {min }}\right) \oplus \widetilde{\mathcal{E}}
$$

with $\widetilde{\mathcal{E}}$ given by the right-hand side of (2.11), and

$$
\operatorname{dim} \mathcal{D}\left(A_{\max }\right) / \mathcal{D}\left(A_{\text {min }}\right)=\operatorname{dim} \mathcal{D}\left(\widetilde{A}_{\max }\right) / \mathcal{D}\left(\widetilde{A}_{\min }\right)=\operatorname{dim} \widetilde{\mathcal{E}}
$$

The latter statements are due to [12], Proposition 1.3.11, Corollary 1.3.17. By Proposition 2.7 it therefore suffices to prove that $\mathcal{E} \subset \mathcal{D}\left(A_{\max }\right)$. In fact, we shall show that $\operatorname{im} G_{k}$ belongs to $\mathcal{D}\left(A_{\max }\right)$ for any $k$. Since this is easy to see for $k=0$, we shall only consider the case $k \geq 1$. With the holomorphic Mellin symbols $f_{n}$ from (2.7) write

$$
A=\widetilde{\omega} t^{-\mu} \sum_{j=0}^{\mu-1} t^{j} \mathrm{op}_{M}\left(f_{j}\right)+t^{\mu} A^{\prime}
$$

for a $\mu$-th order cone differential operator $A^{\prime}$. Taking into account that $\mathrm{im} G_{k l}$ is a subset of $\mathcal{H}_{p}^{\infty, \gamma+\mu+\varepsilon-k+l-1}(\mathbb{B})$ we thus obtain for $u \in \mathcal{C}_{\text {comp }}^{\infty}\left(\partial \mathbb{B}^{\wedge}\right)$

$$
A\left(G_{k} u\right) \in \mathcal{H}_{p}^{0, \gamma}(\mathbb{B}) \Longleftrightarrow \widetilde{\omega} \sum_{j=0}^{k} \sum_{l=0}^{k-j} t^{j} \mathrm{op}_{M}\left(f_{j}\right)\left(G_{k l} u\right) \in \mathcal{H}_{p}^{0, \gamma+\mu}(\mathbb{B}) .
$$

Choosing $\widetilde{\omega}$ with $\widetilde{\omega} \omega=\widetilde{\omega}$, using the elementary rule

$$
\mathrm{op}_{M}(f) t^{\sigma} \mathrm{op}_{M}^{\delta}(g)=t^{\sigma} \mathrm{op}_{M}^{\delta}\left(\left(T^{-\sigma} f\right) g\right),
$$

and rearranging the order of summation, we see that $A\left(G_{k} u\right) \in \mathcal{H}_{p}^{0, \gamma}(\mathbb{B})$ if and only if

$$
\widetilde{\omega} \sum_{j=0}^{k} t^{j} \sum_{l=0}^{j}\left(\operatorname{op}_{M}^{\gamma+\mu+\varepsilon-k-1-\frac{n}{2}}\left(\left(T^{-l} f_{j-l}\right) g_{l}\right)-\operatorname{op}_{M}^{\gamma+\mu+\varepsilon-k-\frac{n}{2}}\left(\left(T^{-l} f_{j-l}\right) g_{l}\right)\right)(u) \in \mathcal{H}_{p}^{0, \gamma+\mu}(\mathbb{B}) \text {. }
$$


However, this expression actually equals zero, since by definition of the symbols $g_{l}$, cf. (2.8), we have

$$
\sum_{l=0}^{j}\left(T^{-l} f_{j-l}\right) g_{l}=\delta_{0 j}, \quad 0 \leq j \leq k,
$$

with $\delta_{0 j}$ denoting the Kronecker symbol. This shows the claim.

Let us turn to the remaining claims of the theorem. If $A$ has constant coefficients near the boundary the intersection of $\mathcal{D}\left(A_{\min }\right)$ and $\mathcal{E}$ is zero by (2.11). Using the description of elements $u$ from $\mathcal{E}$ given in $(2.9)$, and the fact that $\mathcal{D}\left(A_{\text {min }}\right) \subset \mathcal{H}_{p}^{0, \gamma+\mu-\delta}(\mathbb{B})$ for any positive $\delta$, we see that if $u \in \mathcal{D}\left(A_{\text {min }}\right) \cap \mathcal{E}$ then $u$ is of the form (2.9) with $\operatorname{Re} q_{j}=\frac{n+1}{2}-\gamma-\mu$. Let $v:=t^{-\mu} \operatorname{op}_{M}\left(\sigma_{M}^{\mu}(A)\right)(u)$. Inserting the form (2.9), the fact that $v \in \mathcal{H}_{p}^{0, \gamma}(\mathbb{B})$, implies that $v \in \mathcal{C}_{\mathrm{comp}}^{\infty}(\operatorname{int} \mathbb{B})$, in particular, $\widetilde{\omega} v=0$ for a suitable cut-off function $\widetilde{\omega}$. We now set $B_{ \pm \varepsilon}=\operatorname{op}_{M}^{\gamma+\mu \pm \varepsilon-\frac{n}{2}}\left(\sigma_{M}^{\mu}(A)^{-1}\right) t^{\mu}$ for small $\varepsilon>0$. Then

$$
0=B_{-\varepsilon}(\widetilde{\omega} v)=B_{-\varepsilon} v+B_{-\varepsilon}((1-\widetilde{\omega}) v)=u+\left(B_{-\varepsilon}-B_{\varepsilon}\right)((1-\widetilde{\omega}) v)+B_{\varepsilon}((1-\widetilde{\omega}) v) .
$$

We conclude from Lemma 2.4 that the last term is zero and $u \in \operatorname{im}\left(B_{-\varepsilon}-B_{\varepsilon}\right)$. In particular, the intersection $\mathcal{D}\left(A_{\min }\right) \cap \mathcal{E}$ is trivial if $\sigma_{M}^{\mu}(A)^{-1}$ has no pole on the line $\operatorname{Re} z=\frac{n+1}{2}-\gamma-\mu$. This then also proves the last claim of Proposition 2.7 as we announced in the previous proof.

Since $\mathcal{E}$ is finite-dimensional all the operators $\underline{A}$ in $(2.12)$ are in fact closed extensions of $A$.

From Theorem 2.8 and Proposition 2.3 one might conjecture that

$$
\mathcal{D}\left(A_{\min }\right)=\mathcal{H}_{p}^{\mu, \gamma+\mu}(\mathbb{B}) \oplus \operatorname{im} \omega\left(\mathrm{op}_{M}^{\gamma+\mu-\varepsilon-\frac{n}{2}}\left(\sigma_{M}^{\mu}(A)^{-1}\right)-\mathrm{op}_{M}^{\gamma+\mu+\varepsilon-\frac{n}{2}}\left(\sigma_{M}^{\mu}(A)^{-1}\right)\right)
$$

in case $\sigma_{M}^{\mu}(A)^{-1}$ has a pole with real part $\frac{n+1}{2}-\gamma-\mu$. This however is not true, for then $A$ : $\mathcal{H}_{p}^{\mu, \gamma+\mu}(\mathbb{B}) \rightarrow \mathcal{H}_{p}^{0, \gamma}(\mathbb{B})$ would be a Fredholm operator, thus have invertible conormal symbol (by equivalence of ellipticity and Fredholm property in the cone algebra, cf. [20], Theorem 3.13).

Example 2.9. Let $\mathbb{B}$ have dimension 2 and boundary $\partial \mathbb{B}=S^{1}$ (the unit sphere). Define

$$
A=\omega t^{-2}\left\{\frac{1}{4}\left(\left(t \partial_{t}\right)^{2}-t\left(t \partial_{t}\right)\right)+\Delta_{\partial}\right\}+(1-\omega) \Delta,
$$

where $\Delta_{\partial}$ is the standard Laplacian on $S^{1}$ and $\Delta$ some Laplacian on int $\mathbb{B}$. Clearly, A is elliptic; it is $\mathbb{B}$-elliptic, since the rescaled symbol is $-\tau^{2} / 4-|\xi|^{2}$. The conormal symbol

$$
\sigma_{M}^{2}(A)(z)=\frac{1}{4} z^{2}+\Delta_{\partial}
$$

has the non-bijectivity points $z \in 2 \mathbb{Z}$, since the spectrum of $\Delta_{\partial}$ is $\left\{-k^{2} \mid k \in \mathbb{N}_{0}\right\}$.

Considering $A$ as an unbounded operator in $\mathcal{H}_{p}^{0,0}(\mathbb{B})$ we obtain from Proposition 2.3 that $\mathcal{D}\left(A_{\text {min }}\right)=$ $\mathcal{H}_{p}^{2,2}(\mathbb{B})$. From Theorem 2.8 and Proposition 2.7 we conclude that $\mathcal{D}\left(A_{\max }\right)=\mathcal{H}_{p}^{2,2}(\mathbb{B}) \oplus \mathcal{E}$ with a two dimensional space $\mathcal{E}$. Actually, by direct computation,

$$
\mathcal{E}=\operatorname{span}\{\omega, \omega(t+\log t)\} .
$$

In the notation of Definition 2.6, the functions $\omega, \omega \log t$ are generated by $G_{10}$, while $\omega t$ comes from $G_{11}$. The operator $G_{00}$ equals zero, since $\sigma_{M}^{2}(A)^{-1}$ has no poles in the strip $-1 \leq \operatorname{Re} z<0$.

2.4. The model cone operator. Freezing the coefficients of $A$ at the boundary leads to the differential operator

$$
\widehat{A}=t^{-\mu} \sum_{j=0}^{\mu} a_{j}(0)\left(-t \partial_{t}\right)^{j}
$$

on the infinite half-cylinder $\partial \mathbb{B}^{\wedge}=\mathbb{R}_{+} \times \partial \mathbb{B}$. We shall refer to this operator as the model cone operator of $A$. Let us first introduce a suitable scale of Sobolev spaces the operator $\widehat{A}$ acts in.

To this end let $\partial \mathbb{B}=X_{1} \cup \ldots \cup X_{J}$ be an open covering of $\partial \mathbb{B}$; let $\kappa_{j}: X_{j} \rightarrow U_{j}$ be coordinate maps and $\left\{\varphi_{1}, \ldots, \varphi_{J}\right\}$ a subordinate partition of unity. 
Given a function $u=u(t, x)$ on $\mathbb{R} \times \partial \mathbb{B}$, we shall say that $u \in H_{p, \text { cone }}^{s}(\mathbb{R} \times \partial \mathbb{B})$ provided that, for each $j$, the function

$$
v(t, y)=\varphi_{j}(x) u(t, x), \quad x=\kappa_{j}^{-1}(y /[t]),
$$

is an element of $H_{p}^{s}\left(\mathbb{R} \times \mathbb{R}^{n}\right.$ ) (we consider the right-hand side to be zero for $x \notin U_{j}$ ). In other words: $\varphi_{j} u$ is the pull-back of a function in $H_{p}^{s}\left(\mathbb{R}^{n+1}\right)$ under the composition of the maps

$$
\text { id } \times \kappa_{j}: \mathbb{R} \times X_{j} \ni(t, x) \mapsto\left(t, \kappa_{j}(x)\right) \in \mathbb{R} \times U_{j}
$$

and

$$
\chi: \mathbb{R} \times U_{j} \ni(t, y) \mapsto(t,[t] y) \in \mathbb{R}^{n+1},
$$

so that the definition extends to distributions in the usual way for $s \in \mathbb{R}, 1<p<\infty$.

DEFINITION 2.10. $\mathcal{K}_{p}^{s, \gamma}\left(\partial \mathbb{B}^{\wedge}\right)$ is the space of all distributions $u \in H_{p, l o c}^{s}\left(\mathbb{R}_{+} \times \partial \mathbb{B}\right)$ such that, for an arbitrary cut-off function $\omega$,

$$
\omega u \in \mathcal{H}_{p}^{s, \gamma}(\mathbb{B}) \text { and }(1-\omega) u \in H_{p, \text { cone }}^{s}(\mathbb{R} \times \partial \mathbb{B}) .
$$

$\widehat{A}$ acts continuously in this scale of Sobolev spaces,

$$
\widehat{A}: \mathcal{K}_{p}^{s, \gamma}\left(\partial \mathbb{B}^{\wedge}\right) \longrightarrow \mathcal{K}_{p}^{s-\mu, \gamma-\mu}\left(\partial \mathbb{B}^{\wedge}\right), \quad s, \gamma \in \mathbb{R}, 1<p<\infty .
$$

We shall now consider the model cone operator as an unbounded operator, namely

$$
\widehat{A}: \mathcal{C}_{\text {comp }}^{\infty}\left(\partial \mathbb{B}^{\wedge}\right) \subset \mathcal{K}_{p}^{0, \gamma}\left(\partial \mathbb{B}^{\wedge}\right) \longrightarrow \mathcal{K}_{p}^{0, \gamma}\left(\partial \mathbb{B}^{\wedge}\right) .
$$

We first show that the domains of the closed extensions of $\widehat{A}$ can be read off from those of $A$ provided $A$ satisfies a mild additional ellipticity condition. In analogy to Theorems 2.3 and 2.8 we have:

Proposition 2.11. If A satisfies condition (E1) of Section 3.2, then

$$
\mathcal{D}\left(\widehat{A}_{\min }\right)=\left\{u \in \bigcap_{\varepsilon>0} \mathcal{K}_{p}^{\mu, \gamma+\mu-\varepsilon}\left(\partial \mathbb{B}^{\wedge}\right) \mid \widehat{A} u \in \mathcal{K}_{p}^{0, \gamma}(\mathbb{B})\right\}
$$

This simplifies to $\mathcal{D}\left(\widehat{A}_{\min }\right)=\mathcal{K}_{p}^{\mu, \gamma+\mu}\left(\partial \mathbb{B}^{\wedge}\right)$ if and only if $\sigma_{M}^{\mu}(A)$ is invertible on the line $\Gamma_{\frac{n+1}{2}-\gamma-\mu}$. If $\mathcal{E}$ is the space defined in (2.11), then

$$
\mathcal{D}\left(\widehat{A}_{\text {max }}\right)=\mathcal{D}\left(\widehat{A}_{\text {min }}\right) \oplus \mathcal{E}
$$

Hence, any closed extension $\underline{\widehat{A}}$ of $\widehat{A}$ is given by the action of $\widehat{A}$ on a domain

$$
\mathcal{D}(\underline{\widehat{A}})=\mathcal{D}\left(\widehat{A}_{\text {min }}\right) \oplus \underline{\mathcal{E}}, \quad \underline{\mathcal{E}} \text { subspace of } \mathcal{E} .
$$

Proof. Let $\widetilde{P}=\sum_{j=0}^{\mu} a_{j}(0)\left(-\partial_{t}\right)^{j}-\eta^{\mu}$. This is a non-degenerate parameter-dependent differential operator with coefficients independent of $t$. It follows from (E1) in Section 3.2 that the parameter-dependent principal symbol

$$
\widetilde{p}_{0}(x, \xi, \tau, \eta)=\sum_{j=0}^{\mu} \sigma_{\psi}^{\mu-j}\left(a_{j}\right)(0, x, \xi)(-i \tau)^{j}-\eta^{\mu}
$$

of $\widetilde{P}$ is parameter-elliptic. Hence there exist parameter-dependent symbols $\widetilde{q}_{0}$ of order $-\mu$ and $r_{1}, r_{2}$ of order -1 such that

$$
\widetilde{p}_{0} \widetilde{q}_{0}=1+r_{1}, \quad \widetilde{q}_{0} \widetilde{p}_{0}=1+r_{2} .
$$

The operator $P=t^{\mu}\left(\widehat{A}-\eta^{\mu}\right)$ has the principal symbol

$$
\sigma_{\psi}^{\mu}(P)(x, t, \xi, \tau, \eta)=\widetilde{p}_{0}(x, \xi, t \tau, t \eta)
$$

Under the push-forward induced by $T:=\chi \circ\left(\mathrm{id} \times \kappa_{j}\right)$ the operator $P$ transforms into a weighted $S G$-pseudodifferential operator of order $(\mu, \mu)$; modulo terms of order $(\mu-1, \mu-1)$ its $S G$-symbol is given by the push-forward of $\sigma_{\psi}^{\mu}(P)$. Indeed, for a differential operator this is a simple calculation, 
a proof for the general pseudodifferential case is given in 19 , Theorem 3.8; details on $S G$-symbols can be found in $\mathbf{1 8}$. Now equation (2.15) implies that the push-forward of $\sigma_{\psi}^{\mu}(P)$ is $S G$-parameterelliptic if we restrict to a subset of $\mathbb{R}_{+} \times \partial \mathbb{B}$ away from the boundary, say to $\{t \geq 1\}$. Hence, on this set, there is a parameter-dependent $S G$-parametrix $S$ of order $(-\mu,-\mu)$ to the push-forward $T_{*} P$ of $P$ (i.e. we have $S \circ T_{*} P=I+R$, where $R$ is an integral operator with a rapidly decreasing kernel). As the operator of multiplication by $t^{\mu}$ remains unchanged under the push-forward and is an $S G$-operator of order $(0, \mu), S \circ t^{\mu}$ is an $S G$-parametrix of order $(-\mu, 0)$ to the push-forward of $A-\eta^{\mu}$.

We now can describe the domain away from the boundary: Given $u \in \mathcal{K}_{p}^{0, \gamma}$, a cut-off function $\omega$ equal to 1 in $\{t \leq 1\}$, and a function $\varphi_{j}$ in the partition of unity on $\partial \mathbb{B}$,

$$
S \circ t^{\mu} \circ T_{*}\left(\left(\widehat{A}-\eta^{\mu}\right)(1-\omega) \varphi_{j} u\right)=T_{*}(1-\omega) \varphi_{j} u+R T_{*}(1-\omega) \varphi_{j} u
$$

Let $u \in \mathcal{D}\left(\widehat{A}_{\max }\right)$, so that $\widehat{A} u \in \mathcal{K}_{p}^{0, \gamma}$. Standard elliptic regularity implies that $u \in H_{p \text {,loc }}^{\mu}$. Hence $\widehat{A}(1-\omega) \varphi_{j} u \in \mathcal{K}_{p}^{0, \gamma}$ : Outside a compact set, it coincides with the function $(1-\omega) \varphi_{j} \widehat{A} u$ whose push-forward via $T$ belongs to $H_{p}^{0}\left(\mathbb{R}^{n+1}\right)$. In view of the fact that $S t^{\mu}: H_{p}^{0}\left(\mathbb{R}^{n+1}\right) \rightarrow H_{p}^{\mu}\left(\mathbb{R}^{n+1}\right)$ is bounded, we deduce from (2.16) that $(1-\omega) u \in H_{p \text {,cone }}^{\mu}(\mathbb{R} \times \partial \mathbb{B})$. On the other hand, we trivially have $u \in \mathcal{D}\left(\widehat{A}_{\text {min }}\right)$ for every $u$ in $H_{p \text {,cone }}^{\mu}(\mathbb{R} \times \partial \mathbb{B})$ supported in $\{t \geq 1\}$. As a consequence, the domains of all extensions of $\widehat{A}$ coincide with $H_{p, \text { cone }}^{\mu}(\mathbb{R} \times \partial \mathbb{B})$ away from $\{t=0\}$.

Close to $\{t=0\}$, the analysis is the same as in the standard case. This completes the argument.

\section{Structure of the resolvent}

Let us now come to the main objective of this paper. We shall consider a closed extension of a cone differential operator and give conditions that ensure that its resolvent exists in a given sector $\Lambda$ (up to finitely many exceptional points). We shall describe the structure of this resolvent in terms of a class of parameter-dependent cone pseudodifferential operators.

Before considering the resolvent, we want to investigate the inverse of a given closed extension. This is a simpler problem but already illustrates some of the structures we shall see in the discussion of the resolvent. We again refer to [27] and the appendix for basic notions of the cone calculus.

3.1. The inverse of a closed extension. Let $A$ be a cone differential operator and assume that

$$
\underline{A}: \mathcal{D}(\underline{A})=\mathcal{H}_{p}^{\mu, \gamma+\mu}(\mathbb{B}) \oplus \underline{\mathcal{E}} \longrightarrow \mathcal{H}_{p}^{0, \gamma}(\mathbb{B})
$$

is bijective for a fixed choice of $\gamma$ and $p$. Since $\underline{\mathcal{E}}$ is finite-dimensional, $A: \mathcal{H}_{p}^{\mu, \gamma+\mu}(\mathbb{B}) \rightarrow \mathcal{H}_{p}^{0, \gamma}(\mathbb{B})$ is a Fredholm operator. According to [20], Theorem 3.13, this is equivalent to the ellipticity of $A$, i.e. $A$ is $\mathbb{B}$-elliptic and the conormal symbol is invertible on the line $\Gamma_{\frac{n+1}{2}-\gamma-\mu}$. In other words, $A$ is an elliptic element of the cone algebra $C^{\mu}(\gamma+\mu, \gamma, k)$ for any $k \in \mathbb{N}$.

Let us now set $\underline{\mathcal{F}}=A(\underline{\mathcal{E}})$. This space is finite-dimensional and $\mathcal{H}_{p}^{0, \gamma}(\mathbb{B})=A\left(\mathcal{H}_{p}^{\mu, \gamma+\mu}(\mathbb{B})\right) \oplus \underline{\mathcal{F}}$.

LEMmA 3.1. There exists an asymptotic type $Q \in \operatorname{As}(\gamma, k), k \in \mathbb{N}$ arbitrarily large, such that $\underline{\mathcal{F}}=A(\underline{\mathcal{E}}) \subset \mathcal{C}_{Q}^{\infty, \gamma}(\mathbb{B})$.

Proof. Let $u$ be of the form (2.9), and choose a cut-off function $\widetilde{\omega}$ with $\widetilde{\omega} \omega=\widetilde{\omega}$. Then, with $A$ as in (2.1),

$$
(A u)(t)=\widetilde{\omega} t^{-\mu} \sum_{j=0}^{\mu} \sum_{q, k} a_{j}(t)\left(t \partial_{t}\right)^{j}\left(c_{q k} t^{-q} \log ^{k} t\right)+(1-\widetilde{\omega})(t)(A u)(t) .
$$

The second term belongs to $\mathcal{C}_{\text {comp }}^{\infty}($ int $\mathbb{B})$. Now a Taylor expansion of the coefficients $a_{j}$ in $t$ at 0 shows the claim. 
Let us now denote by

$$
\pi_{\underline{\mathcal{E}}}: \mathcal{H}_{p}^{\mu, \gamma+\mu}(\mathbb{B}) \oplus \underline{\mathcal{E}} \longrightarrow \underline{\mathcal{E}}, \quad \pi_{\mathcal{F}}: A\left(\mathcal{H}_{p}^{\mu, \gamma+\mu}(\mathbb{B})\right) \oplus \underline{\mathcal{F}} \longrightarrow \underline{\mathcal{F}}
$$

the canonical projections, and let

$$
B=\left(1-\pi_{\underline{\mathcal{E}}}\right) \underline{A}^{-1}: \mathcal{H}_{p}^{0, \gamma}(\mathbb{B}) \longrightarrow \mathcal{H}_{p}^{\mu, \gamma+\mu}(\mathbb{B})
$$

be a left-inverse of $A: \mathcal{H}_{p}^{\mu, \gamma+\mu}(\mathbb{B}) \rightarrow \mathcal{H}_{p}^{0, \gamma}(\mathbb{B})$.

LEMMA 3.2. $1-A B=\pi_{\underline{\mathcal{F}}}$ belongs to $C_{G}(\gamma, \gamma, k)$ for arbitrarily large $k \in \mathbb{N}$.

Proof. By construction, it is clear that $1-A B=\pi_{\mathcal{F}}$. Let $w_{1}, \ldots, w_{N}$ be a basis of $\underline{\mathcal{F}}$. Then we can write $\pi_{\mathcal{F}}(\cdot)=\sum_{j=1}^{N} l_{j}(\cdot) w_{j}$ with functionals $l_{j}$ on $\mathcal{H}_{p}^{0, \gamma}(\mathbb{B})$. By duality there exist $v_{j} \in \mathcal{H}_{p^{\prime}}^{0,-\gamma}(\mathbb{B})$ such that $l_{j}(\cdot)=\left\langle\cdot, v_{j}\right\rangle_{\mathcal{H}_{2}^{0,0}(\mathbb{B})}$. Then, for all $u \in \mathcal{H}_{p}^{\mu, \gamma+\mu}(\mathbb{B})$,

$$
0=\pi_{\underline{\mathcal{F}}}(A u)=\sum_{j=1}^{N}\left\langle A u, v_{j}\right\rangle_{\mathcal{H}_{2}^{0,0}(\mathbb{B})} w_{j}=\sum_{j=1}^{N}\left\langle u, A^{*} v_{j}\right\rangle_{\mathcal{H}_{2}^{0,0}(\mathbb{B})} w_{j},
$$

where $A^{*}$ denotes the formal adjoint of $A$, which belongs to $C^{\mu}(-\gamma,-\gamma-\mu, k)$. Hence $v_{j} \in \operatorname{ker} A^{*}$ for $j=1, \ldots, N$. Since with $A$ also $A^{*}$ is an elliptic cone operator, $\operatorname{ker} A^{*} \subset \mathcal{C}_{Q^{\prime}}^{\infty,-\gamma}(\mathbb{B})$ for some asymptotic type $Q^{\prime} \in \operatorname{As}(-\gamma, k)$ by elliptic regularity, cf. [22], Theorem 8 in Section 2.2.1. Thus $\pi_{\mathcal{F}}$ has a kernel in $\underline{\mathcal{F}} \otimes \mathcal{C}_{Q^{\prime}}^{\infty},-\gamma(\mathbb{B})$, and therefore is a Green operator.

Proposition 3.3. $B$ is an element of $C^{-\mu}(\gamma, \gamma+\mu, k)$ for arbitrarily large $k \in \mathbb{N}$.

Proof. Since $A$ is an elliptic element of $C^{\mu}(\gamma+\mu, \gamma, k)$ as we have seen above, there exists a parametrix $C \in C^{-\mu}(\gamma, \gamma+\mu, k)$, i.e.

$$
A C-1=G_{R} \in C_{G}(\gamma, \gamma, k), \quad C A-1=G_{L} \in C_{G}(\gamma+\mu, \gamma+\mu, k) .
$$

Multiplying these identities from the left, respectively from the right with $B$ yields $B=C-B G_{R}$ and $B=C A B-G_{L} B=C-C(1-A B)-G_{L} B$. Inserting the first equation into the second gives

$$
B=C-C \pi_{\underline{\mathcal{F}}}-G_{L} C+G_{L} B G_{R} .
$$

The third term on the right-hand side is a Green operator, since these form an ideal in the cone algebra. The same is true for the second term in view of Lemma 3.2 and for the forth due to the continuity of $B$ and the mapping properties of Green operators.

ThEOREM 3.4. $\underline{A}^{-1}=B+G$ for the above $B \in C^{-\mu}(\gamma, \gamma+\mu, k)$ and a suitable $G \in C_{G}(\gamma, \gamma, k)$ with arbitrarily large $k \in \mathbb{N}$.

Proof. We decompose $\underline{A}^{-1}=\left(1-\pi_{\underline{\mathcal{E}}}\right) \underline{A}^{-1}+\underline{A}^{-1} \pi_{\mathcal{F}}=B+G$. By Proposition 3.3 , $B$ is as claimed. From the proof of Lemma 3.2 we know that $\pi_{\underline{\mathcal{F}}}$ has an integral kernel in $\underline{\mathcal{F}} \otimes \mathcal{C}_{Q^{\prime}}^{\infty,-\gamma}(\mathbb{B})$ for some type $Q^{\prime} \in \operatorname{As}(-\gamma, k)$. Therefore, $\underline{A}^{-1} \pi_{\underline{\mathcal{F}}}$ has a kernel in $\underline{\mathcal{E}} \otimes \mathcal{C}_{Q^{\prime}}^{\infty,-\gamma}(\mathbb{B})$, hence is a Green operator.

As we shall explain in Section 4.1, the invertibility is independent of $1<p<\infty$.

3.2. Ellipticity assumptions and resolvent analysis. Let $A$ be a cone differential operator of order $\mu$ whose coefficients on $[0,1] \times \partial \mathbb{B}$ are independent of $t$ and let

$$
\underline{A}: \mathcal{D}(\underline{A})=\mathcal{D}\left(A_{\min }\right) \oplus \underline{\mathcal{E}} \subset \mathcal{H}_{p}^{0, \gamma}(\mathbb{B}) \longrightarrow \mathcal{H}_{p}^{0, \gamma}(\mathbb{B})
$$

be a closed extension. Moreover, we assume

(E1) Both $\sigma_{\psi}^{\mu}(A)$ and $\widetilde{\sigma}_{\psi}^{\mu}(A)$ have no spectrum in the sector $\Lambda$,

(E2) With the above choice of $\underline{\mathcal{E}}$, the domain $\mathcal{D}(\underline{\widehat{A}})=\mathcal{D}\left(\widehat{A}_{\min }\right) \oplus \underline{\mathcal{E}}$ of the model cone operator is invariant under dilations, 
(E3) The sector $\Lambda \backslash\{0\}$ contains no spectrum of the model cone operator

$$
\underline{\widehat{A}}: \mathcal{D}(\underline{\widehat{A}}) \subset \mathcal{K}_{p}^{0, \gamma}\left(\partial \mathbb{B}^{\wedge}\right) \longrightarrow \mathcal{K}_{p}^{0, \gamma}\left(\partial \mathbb{B}^{\wedge}\right) .
$$

In (E1) and (E3), $\Lambda=\Lambda_{\theta}$ is a closed sector in the complex plane containing zero, i.e.

$$
\Lambda_{\theta}=\{z \in \mathbb{C}|| \arg z \mid \geq \theta\} \cup\{0\},
$$

where $0 \leq \theta<\pi$ and $-\pi \leq \arg z<\pi$.

In order to simplify the exposition, we fix a sector $\Sigma$ such that the mapping $\eta \mapsto \eta^{\mu}$ induces a bijection $\Sigma \rightarrow \Lambda$. Instead of considering $\lambda-A$ for $\lambda \in \Lambda$ we then study $\eta^{\mu}-A$ for $\eta \in \Sigma$.

Condition (E2) means the following: Whenever $u=u(t, x)$ belongs to $\mathcal{D}(\underline{\widehat{A}})$, the same is true for the functions $u_{\varrho}(t, x)=u(\varrho t, x), \varrho>0$. It is easy to see that the domain $\mathcal{D}(\underline{\widehat{A}})$ is invariant under dilations if and only if this is true for $\mathcal{C}_{\text {comp }}^{\infty}\left(\partial \mathbb{B}^{\wedge}\right) \oplus \underline{\mathcal{E}}$. Gil and Mendoza 10 call such a domain saturated. Condition (E2) is always satisfied for the minimal extension, the maximal extension, and for extensions with domain equal to $\mathcal{D}\left(A_{\max }\right) \cap \mathcal{H}_{p}^{0, \sigma}(\mathbb{B})$ and $\gamma-\mu<\sigma \leq \gamma$. For concrete examples see Section 5 below.

THEOREM 3.5. Let $\underline{A}$ satisfy conditions (E1), (E2), and (E3) with respect to the sector $\Lambda$. Then

a) $\underline{A}$ has at most finitely many spectral points in $\Lambda$.

b) There exists a parameter-dependent cone pseudodifferential operator

$$
c(\eta) \in C_{O}^{-\mu}(\Sigma)+C_{G}^{-\mu}(\Sigma ; \gamma, \gamma, \varepsilon)
$$

with a certain $\varepsilon>0$, such that $\left(\eta^{\mu}-\underline{A}\right)^{-1}=c(\eta)$ for $\eta \in \Sigma$ with $|\eta|$ sufficiently large.

For the notation used in part b) of this theorem we refer to the appendix (see Definition 6.6 and Definition 6.13). Part a) of the theorem follows from b): Since the domain of $\underline{A}$ is compactly embedded in $\mathcal{H}_{p}^{0, \gamma}(\mathbb{B}), \underline{A}$ has a compact resolvent, hence a discrete spectrum.

The proof of Theorem 3.5 is the most technical part of the paper, and it relies on the material in the appendix. The basic idea is as follows: We write $a(\eta)=\eta^{\mu}-A$. This is a parameter-dependent family of cone differential operators of order $\mu$, i.e.,

$$
a(\eta) \in C_{O}^{\mu}(\Sigma) \subset C^{\mu}(\Sigma ; \widetilde{\gamma}, \widetilde{\gamma}-\mu, k)
$$

for any $\widetilde{\gamma} \in \mathbb{R}$ and $k \in \mathbb{N}$. Assumption (E1) ensures that $a(\eta)$ is $\mathbb{B}$-elliptic with respect to the sector $\Sigma$. Hence the conormal symbol of $a(\eta)$ (which is that of $-A$ ) is meromorphically invertible as shown in 22], 2.2.1, Theorem 14 and 2.3.1, Theorem 16. As we do not require $A$ to be conormally elliptic with respect to the weight $\gamma+\mu$, the inverse might have a pole on the line $\{\operatorname{Re} z=$ $\left.\frac{n+1}{2}-\gamma-\mu\right\}$; we fix $\varepsilon_{0}>0$ such that there exists no pole with real part between $\frac{n+1}{2}-\gamma-\mu$ and $\frac{n+1}{2}-\gamma-\mu \pm 2 \varepsilon_{0}$. In Propositions 3.6 and 3.7, we next construct right and left parametrices modulo parameter-dependent zero order Green operators. Using conditions (E2) and (E3) we then can express $\left(\eta^{\mu}-\underline{\widehat{A}}\right)^{-1}$ as a principal edge symbol, and finally understand $\left(\eta^{\mu}-\underline{A}\right)^{-1}$.

Proposition 3.6. For any $0<\varepsilon<\varepsilon_{0}$ there exists a parameter-dependent cone operator $b_{R}(\eta) \in$ $C^{-\mu}(\Sigma ; \gamma-\varepsilon, \gamma+\mu-\varepsilon, \mu)$ such that

$$
a(\eta) b_{R}(\eta)-1=g_{R}(\eta) \in C_{G}^{0}(\Sigma ; \gamma-\varepsilon, 1-2 \varepsilon, O ; \gamma+\varepsilon, 1-2 \varepsilon, Q)
$$

with a suitable asymptotic type $Q \in \operatorname{As}(\gamma+\varepsilon, 1-2 \varepsilon)$. Moreover, for each $\eta$, the action of $b_{R}(\eta)$ on $\mathcal{H}_{p}^{0, \gamma}(\mathbb{B})$ is independent of $\varepsilon$ and yields a bounded mapping

$$
b_{R}(\eta): \mathcal{H}_{p}^{0, \gamma}(\mathbb{B}) \longrightarrow \mathcal{D}\left(A_{\min }\right) .
$$

Proof. According to Theorem 6.8 there exists a flat parameter-dependent cone operator $\widetilde{b}(\eta) \in C_{O}^{-\mu}(\Sigma)$ such that

$$
a(\eta) \widetilde{b}(\eta)-1 \equiv \omega(t[\eta]) \operatorname{op}_{M}(\widetilde{f})(\eta) \omega_{0}(t[\eta]) \quad \bmod C_{G}^{0}(\Sigma)_{\infty}
$$


with a holomorphic Mellin symbol $\widetilde{f} \in M^{-\infty}(\partial \mathbb{B} ; \Sigma)$. Setting $\widetilde{f}_{0}(z)=\widetilde{f}(z, 0)$, we have

$$
\omega(t[\eta])\left\{\operatorname{op}_{M}^{\tilde{\gamma}-\frac{n}{2}}(\widetilde{f})(\eta)-\operatorname{op}_{M}^{\widetilde{\gamma}-\frac{n}{2}}\left(\widetilde{f}_{0}\right)\right\} \omega_{0}(t[\eta]) \in C_{G}^{0}(\Sigma ; \widetilde{\gamma}, 1, O ; \widetilde{\gamma}, 1, O)
$$

for any $\widetilde{\gamma} \in \mathbb{R}$. In fact, by a Taylor expansion, $\widetilde{f}(z, t \eta)=\widetilde{f}_{0}(z)+\sum_{i=1}^{n}\left(t \eta_{i}\right) \tilde{f}_{i}(z, t \eta)$ for suitable $\widetilde{f}_{i}(z, \eta) \in \mathcal{C}^{\infty}\left(\Sigma, M_{O}^{-\infty}(\partial \mathbb{B})\right)$. Therefore, the operator-family in (3.3) pointwise has the mapping properties of Green symbols from $R_{G}^{0}(\Sigma ; \widetilde{\gamma}, 1, O ; \widetilde{\gamma}, 1, O)$, cf. Definition 6.11. Moreover, it is homogeneous of order 0 for large $|\eta|$ in the sense of (6.9) with respect to the group action of (6.10), hence also satisfies the required symbol estimates.

Next, we are going to modify $\widetilde{b}(\eta)$ by a smoothing Mellin term, i.e. we set

$$
b_{R}(\eta)=\widetilde{b}(\eta)+\omega(t[\eta]) t^{\mu} \operatorname{op}_{M}^{\gamma-\varepsilon-\frac{n}{2}}(f) \omega_{0}(t[\eta])
$$

where we determine $f$ in such a way that the conormal symbol of $a(\eta) b_{R}(\eta)-1$ vanishes, i.e.

$$
0=\sigma_{M}^{0}\left(a b_{R}\right)(z)-1=\sigma_{M}^{0}(a \widetilde{b})(z)+\sigma_{M}^{\mu}(a)(z-\mu) f(z)-1=\widetilde{f}_{0}(z)+\sigma_{M}^{\mu}(a)(z-\mu) f(z) .
$$

Solving for $f$ yields

$$
f(z)=-\sigma_{M}^{\mu}(a)^{-1}(z-\mu) \widetilde{f}_{0}(z)=\sigma_{M}^{\mu}(a)^{-1}(z-\mu)-\sigma_{M}^{-\mu}(\widetilde{b})(z) .
$$

By our choice of $\varepsilon_{0}$ in connection with Lemma 2.4, the action of $b_{R}(\eta)$ on $\mathcal{H}_{p}^{s, \gamma}(\mathbb{B})$ is independent of $\varepsilon$. Moreover, the description of $\mathcal{D}\left(A_{\min }\right)$ given in Proposition 2.3 together with (3.5) implies that $b_{R}(\eta)$ maps $\mathcal{H}_{p}^{0, \gamma}(\mathbb{B})$ to $\mathcal{D}\left(A_{\min }\right)$. Choose a cut-off function $\omega_{1}$ with $\omega \omega_{1}=\omega_{1}$. We claim that

$$
a(\eta) b_{R}(\eta)-1 \equiv \omega_{1}(t[\eta]) \operatorname{op}_{M}^{\gamma-\varepsilon-\frac{n}{2}}\left(\widetilde{f}_{0}\right) \omega_{0}(t[\eta])+\omega_{1}(t[\eta]) a(\eta) \omega(t[\eta]) t^{\mu} \operatorname{op}_{M}^{\gamma-\varepsilon-\frac{n}{2}}(f) \omega_{0}(t[\eta])
$$

modulo $C_{G}^{0}(\Sigma ; \gamma-\varepsilon, 1-2 \varepsilon, O ; \gamma+\varepsilon, 1-2 \varepsilon, O)$. Indeed, this follows from (3.2) and (3.3) together with the fact that changing $\omega$ in $(3.2)$ to $\omega_{1}$ only causes a remainder in $C_{G}^{0}(\Sigma)_{\infty}$, and Lemma 6.14 (for $\gamma-\varepsilon$ instead of $\gamma$ ).

Since $a(\eta)=\eta^{\mu}-t^{-\mu} \mathrm{Op}_{M}\left(\sigma_{M}^{\mu}(a)\right)$ on $[0,1] \times \partial \mathbb{B}$, an application of (2.13) shows that the second term on the right-hand side of (3.6) equals

$$
\begin{aligned}
& -\omega_{1}(t[\eta]) \operatorname{op}_{M}^{\gamma-\varepsilon-\frac{n}{2}}\left(\widetilde{f}_{0}\right) \omega_{0}(t[\eta])+\omega_{1}(t[\eta])(t \eta)^{\mu} \operatorname{op}_{M}^{\gamma-\varepsilon-\frac{n}{2}}(f) \omega_{0}(t[\eta]) \\
& -\omega_{1}(t[\eta]) \operatorname{op}_{M}^{\gamma-\varepsilon-\frac{n}{2}}\left(T^{-\mu} \sigma_{M}^{\mu}(a)\right)(1-\omega)(t[\eta]) \operatorname{op}_{M}^{\gamma-\varepsilon-\frac{n}{2}}(f) \omega_{0}(t[\eta]) .
\end{aligned}
$$

The first term cancels with the first term of (3.6), the other two belong to $C_{G}^{0}(\Sigma ; \gamma-\varepsilon, 1, O ; \gamma+$ $\varepsilon, 1, Q)$, where $Q$ is the asymptotic type induced by the meromorphic structure of $\left(T^{-\mu} \sigma_{M}^{\mu}(a)^{-1}\right)^{*}$.

In a similar way, one can also construct a rough left-parametrix for $a(\eta)$ :

Proposition 3.7. Let $0<\varepsilon<\varepsilon_{0}$. Then there exists a parameter-dependent cone operator $b_{L}(\eta) \in$ $C^{-\mu}(\Sigma ; \gamma-\mu+\varepsilon, \gamma+\varepsilon, \mu)$ such that

$$
b_{L}(\eta) a(\eta)-1=g_{L}(\eta) \in C_{G}^{0}(\Sigma ; \gamma+\varepsilon, \gamma+\varepsilon, \mu) .
$$

Passing in (3.1), (3.7) to the principal edge symbols, cf. (6.16), and solving for $\left(\eta^{\mu}-\underline{\widehat{A}}\right)^{-1}$ yields

$$
\left(\eta^{\mu}-\underline{\widehat{A}}\right)^{-1}=\sigma_{\wedge}^{-\mu}\left(b_{R}\right)(\eta)-\sigma_{\wedge}^{-\mu}\left(b_{L}\right)(\eta) \sigma_{\wedge}^{0}\left(g_{R}\right)(\eta)+\sigma_{\wedge}^{0}\left(g_{L}\right)(\eta)\left(\eta^{\mu}-\underline{\widehat{A}}\right)^{-1} \sigma_{\wedge}^{0}\left(g_{R}\right)(\eta)
$$

on $\mathcal{K}_{p}^{0, \gamma}\left(\partial \mathbb{B}^{\wedge}\right)$. We are now going to show that the second and third term on the right-hand side of (3.8) are the principal edge symbol of a parameter-dependent Green operator. Let us set

$$
\underline{\mathcal{E}}^{\prime}=\underline{\mathcal{E}} \oplus \operatorname{im} \omega\left(\operatorname{op}_{M}^{\gamma+\mu-\varepsilon-\frac{n}{2}}\left(\sigma_{M}^{\mu}(A)^{-1}\right)-\mathrm{op}_{M}^{\gamma+\mu+\varepsilon-\frac{n}{2}}\left(\sigma_{M}^{\mu}(A)^{-1}\right)\right)
$$

i.e. we add to $\underline{\mathcal{E}}$ the asymptotic terms coming from the poles of $\sigma_{M}^{\mu}(A)^{-1}$ on the line $\operatorname{Re} z=$ $\frac{n+1}{2}-\gamma-\mu$. Note that these terms belong to $\mathcal{D}\left(A_{\min }\right)$. 
Proposition 3.8. There exists a Green symbol $g \in R_{G}^{-\mu}(\Sigma ; \gamma, \varepsilon ; \gamma, \mu+\varepsilon)$ for some $\varepsilon>0$ such that

$$
\sigma_{\wedge}^{-\mu}(g)(\eta)=-\sigma_{\wedge}^{-\mu}\left(b_{L}\right)(\eta) \sigma_{\wedge}^{0}\left(g_{R}\right)(\eta)+\sigma_{\wedge}^{0}\left(g_{L}\right)(\eta)\left(\eta^{\mu}-\underline{\widehat{A}}\right)^{-1} \sigma_{\wedge}^{0}\left(g_{R}\right)(\eta)
$$

and the integral kernel $k_{g}$ of $g$, cf. (6.1) and Theorem 6.12, satisfies

$$
k_{g}\left(\eta, t, x, t^{\prime}, x^{\prime}\right) \in S_{c l}^{-\mu}\left(\Sigma, \mathcal{S}_{\underline{\mathcal{E}}^{\prime}}^{\gamma}\left(\partial \mathbb{B}^{\wedge}\right) \widehat{\otimes}_{\pi} \mathcal{S}_{\varepsilon / 2}^{-\gamma}\left(\partial \mathbb{B}^{\wedge}\right)\right)=S_{c l}^{-\mu}(\Sigma) \widehat{\otimes}_{\pi} \mathcal{S}_{\underline{\mathcal{E}}^{\prime}}^{\gamma}\left(\partial \mathbb{B}^{\wedge}\right) \widehat{\otimes}_{\pi} \mathcal{S}_{\varepsilon / 2}^{-\gamma}\left(\partial \mathbb{B}^{\wedge}\right),
$$

where we set $\mathcal{S}_{\underline{\mathcal{E}}^{\prime}}^{\gamma}\left(\partial \mathbb{B}^{\wedge}\right)=\mathcal{S}_{\varepsilon / 2}^{\gamma+\mu}\left(\partial \mathbb{B}^{\wedge}\right) \oplus \underline{\mathcal{E}}^{\prime}$.

ProOF. For brevity let us write $\widehat{g}(\eta)=\sigma_{\wedge}^{-\mu}(g)(\eta)$, and analogously for the principal edge symbols of $b, g_{L}$, and $g_{R}$. By the previous proposition, we have

$$
\mathcal{K}_{2}^{s, \gamma}\left(\partial \mathbb{B}^{\wedge}\right) \hookrightarrow \mathcal{K}_{2}^{s, \gamma-\varepsilon}\left(\partial \mathbb{B}^{\wedge}\right) \stackrel{\widehat{g}_{R}(\eta)}{\longrightarrow} \mathcal{S}_{Q}^{\gamma+\varepsilon}\left(\partial \mathbb{B}^{\wedge}\right) \hookrightarrow \mathcal{S}_{Q}^{\gamma-\mu+\varepsilon}\left(\partial \mathbb{B}^{\wedge}\right)
$$

where one considers $Q \in \operatorname{As}(\gamma+\varepsilon, 1-2 \varepsilon)$ as an asymptotic type $Q \in \operatorname{As}(\gamma-\mu+\varepsilon, \mu)$ for the last embedding. By standard mapping properties of cone operators there exists an asymptotic type $Q^{\prime} \in \operatorname{As}(\gamma+\varepsilon, \mu)$ such that $\widehat{b}_{L}(\eta): \mathcal{S}_{Q}^{\gamma-\mu+\varepsilon}\left(\partial \mathbb{B}^{\wedge}\right) \rightarrow \mathcal{S}_{Q^{\prime}}^{\gamma+\varepsilon}\left(\partial \mathbb{B}^{\wedge}\right) \hookrightarrow \mathcal{S}_{Q^{\prime}}^{\gamma}\left(\partial \mathbb{B}^{\wedge}\right)$, considering $Q^{\prime}$ as an element of $\operatorname{As}(\gamma, \mu+\varepsilon)$. Making similar considerations for the adjoint, we thus obtain $\widehat{b}_{L}(\eta) \widehat{g}_{R}(\eta) \in R_{G}^{(-\mu)}(\Sigma ; \gamma, \varepsilon ; \gamma, \mu+\varepsilon)$.

Next, $\left(\eta^{\mu}-\underline{\widehat{A}}\right)^{-1}$ is a smooth function on $\Sigma \backslash\{0\}$ with values in $\mathcal{L}\left(\mathcal{K}_{p}^{0, \gamma}\left(\partial \mathbb{B}^{\wedge}\right), \mathcal{D}(\underline{\widehat{A}})\right)$. In view of assumption (E2) on the scaling invariance of $\underline{\mathcal{E}}$, it is homogeneous of order $-\mu$ in the sense of $(6.9)$. In particular, $\left(\eta^{\mu}-\underline{\widehat{A}}\right)^{-1} \in S^{(-\mu)}\left(\Sigma ; \mathcal{K}_{p}^{0, \gamma}\left(\partial \mathbb{B}^{\wedge}\right), \mathcal{K}_{p}^{0, \gamma+\varepsilon}\left(\partial \mathbb{B}^{\wedge}\right)\right.$ for sufficiently small $\varepsilon>0$. But then it is clear that $\widehat{g}_{L}(\eta)\left(\eta^{\mu}-\underline{\widehat{A}}\right)^{-1} \widehat{g}_{R}(\eta)$ also belongs to $R_{G}^{(-\mu)}(\Sigma ; \gamma, \varepsilon ; \gamma, \mu+\varepsilon)$. If we now define

$$
g(\eta)=\chi(\eta)\left\{-\widehat{b}_{L}(\eta) \widehat{g}_{R}(\eta)+\widehat{g}_{L}(\eta)\left(\eta^{\mu}-\underline{\widehat{A}}\right)^{-1} \widehat{g}_{R}(\eta)\right\}
$$

with an arbitrary zero excision function $\chi(\eta)$, then $g \in R_{G}^{-\mu}(\Sigma ; \gamma, \varepsilon ; \gamma, \mu+\varepsilon)$, and the principal edge symbol is given by the formula stated in the proposition. It remains to investigate the kernel.

Since $g(\eta)$ is a Green symbol of the given class (and by the kernel characterization) there exists some asymptotic type $Q \in \operatorname{As}\left(\gamma, \mu+\frac{\varepsilon}{2}\right)$ such that the integral kernel $k_{g}$ of $g(\eta)$ belongs to $S_{c l}^{-\mu}(\Sigma) \widehat{\otimes}_{\pi} \mathcal{S}_{Q}^{\gamma+\varepsilon}\left(\partial \mathbb{B}^{\wedge}\right) \widehat{\otimes}_{\pi} \mathcal{S}_{\varepsilon / 2}^{-\gamma}\left(\partial \mathbb{B}^{\wedge}\right)$. According to Definition 6.10,

$$
\mathcal{S}_{Q}^{\gamma+\varepsilon}\left(\partial \mathbb{B}^{\wedge}\right)=\mathcal{S}_{\mu}^{\gamma+\varepsilon / 2}\left(\partial \mathbb{B}^{\wedge}\right) \oplus \mathcal{E}_{Q}
$$

By possibly shrinking $\varepsilon$, we may assume that $Q$ contains no triple $(q, l, L)$ with $\operatorname{Re} q<\frac{n+1}{2}-\gamma-\mu$. By possibly enlarging $Q$ we can assume that $\underline{\mathcal{E}}^{\prime} \subset \mathcal{E}_{Q}$, and therefore $\mathcal{E}_{Q}=\underline{\mathcal{E}}^{\prime} \oplus V$ for a certain finite-dimensional space $V$. Therefore $k_{g}$ can be written as $k_{g}=k_{g}^{0}+k_{g}^{1}$ with $k_{g}^{0}$ containing the contribution of $\underline{\mathcal{E}}^{\prime}$, and $k_{g}^{1}$ that of $V$. However, from identity (3.8) one sees that $g(\eta)$ maps into the domain of $\underline{\widehat{A}}$, and therefore $k_{g}^{1}$ must equal 0 . This then finishes the proof, since $\mathcal{S}_{\mu}^{\gamma+\varepsilon / 2}\left(\partial \mathbb{B}^{\wedge}\right)=$ $\mathcal{S}_{\varepsilon / 2}^{\gamma+\mu}\left(\partial \mathbb{B}^{\wedge}\right)$.

With $b_{R}(\eta)$ from Proposition 3.6 and $g(\eta)$ from Proposition 3.8 let us now define

$$
b(\eta)=b_{R}(\eta)+\sigma g(\eta) \sigma_{0}=\widetilde{b}(\eta)+\omega(t[\eta]) t^{\mu} \mathrm{op}_{M}^{\gamma-\varepsilon-\frac{n}{2}}(f) \omega_{0}(t[\eta])+\sigma g(\eta) \sigma_{0}
$$

wit cut-off functions $\sigma, \sigma_{0}$. Clearly, $b(\eta): \mathcal{H}_{p}^{0, \gamma}(\mathbb{B}) \rightarrow \mathcal{D}(\underline{A})$ for each $\eta$. As explained in Definition 6.6 and the subsequent paragraph, there are then $h, p$, and a cut-off function $\sigma_{1}$ such that

$$
b(\eta)=\sigma\left\{t^{\mu} \mathrm{op}_{M}^{\gamma-\frac{n}{2}}(h)(\eta)+\omega(t[\eta]) t^{\mu} \mathrm{op}_{M}^{\gamma-\varepsilon-\frac{n}{2}}(f) \omega_{0}(t[\eta])+g(\eta)\right\} \sigma_{0}+(1-\sigma) p(\eta)\left(1-\sigma_{1}\right) .
$$

Since $A$ has $t$-independent coefficients on $[0,1] \times \partial \mathbb{B}$, we can also choose $h$ to be independent of $t$, i.e. $h \in M_{O}^{-\mu}(\partial \mathbb{B} ; \Sigma)$. Then

$$
\sigma_{\wedge}^{-\mu}\left(b_{R}\right)(\eta)=t^{\mu} \operatorname{op}_{M}^{\gamma-\frac{n}{2}}(h)(\eta)+\omega(t|\eta|) t^{\mu} \operatorname{op}_{M}^{\gamma-\varepsilon-\frac{n}{2}}(f) \omega_{0}(t|\eta|) .
$$


As $\sigma_{\wedge}^{-\mu}(g)(\eta)=g(\eta)$ for large $|\eta|$ by construction of, we deduce from (3.8) and (3.11) that

$$
b(\eta)=\sigma\left(\eta^{\mu}-\underline{\widehat{A}}\right)^{-1} \sigma_{0}+(1-\sigma) p(\eta)\left(1-\sigma_{1}\right), \quad|\eta| \geq R,
$$

for a sufficiently large $R>0$. Changing the cut-off functions only alters $b(\eta)$ by a nice remainder:

Lemma 3.9. Let $\widetilde{\sigma}, \widetilde{\sigma}_{0}, \widetilde{\sigma}_{1}$ be cut-off functions satisfying the conditions posed in Definition 6.6 and the subsequent paragraph. Then

$$
b(\eta)=\widetilde{\sigma}\left(\eta^{\mu}-\underline{\widehat{A}}\right)^{-1} \widetilde{\sigma}_{0}+(1-\widetilde{\sigma}) p(\eta)\left(1-\widetilde{\sigma}_{1}\right)+r(\eta), \quad|\eta| \geq R,
$$

with a remainder $r(\eta)$ having an integral kernel (with a certain $\varepsilon>0$ )

$$
k_{r}\left(\eta, y, y^{\prime}\right) \in \mathcal{S}\left(\Sigma, \mathcal{C}_{\underline{\mathcal{E}}^{\prime}}^{\infty, \gamma}(\mathbb{B}) \widehat{\otimes}_{\pi} \mathcal{C}_{\varepsilon}^{\infty,-\gamma}(\mathbb{B})\right) .
$$

Here, we have set $\mathcal{C}_{\underline{\mathcal{E}}^{\prime}}^{\infty, \gamma}(\mathbb{B})=\mathcal{C}_{\varepsilon}^{\infty, \gamma+\mu} \oplus \underline{\mathcal{E}}^{\prime}$. Hence we have not only $r(\eta) \in C_{G}^{-\infty}(\Sigma ; \gamma, \gamma, \varepsilon)$, but also $r(\eta) \in \mathcal{S}\left(\Sigma, \mathcal{L}\left(\mathcal{\mathcal { H }}_{p}^{0}, \gamma(\mathbb{B}), \mathcal{D}(\underline{A})\right)\right)$.

Proof. This statement is easily seen using the representation of $b(\eta)$ in (3.10). Changing cutoff functions alters $\widetilde{b}(\eta)$ only by a flat Green symbol in $C_{G}^{-\infty}(\Sigma)_{\infty}$, which has in particular a kernel of the mentioned structure. It remains to note that $\sigma g(\eta) \sigma_{0}-\widetilde{\sigma} g(\eta) \widetilde{\sigma}_{0}=\sigma g(\eta)\left(\sigma_{0}-\widetilde{\sigma}_{0}\right)+(\widetilde{\sigma}-\sigma) g(\eta) \widetilde{\sigma}_{0}$ and both these terms have the required structure (recall that they are Green symbols of order $-\infty$, since both $\widetilde{\sigma}-\sigma$ and $\sigma_{0}-\widetilde{\sigma}_{0}$ belong to $\left.\mathcal{C}_{\text {comp }}^{\infty}(] 0,1[)\right)$.

Proposition 3.10. If $b(\eta)$ is as in (3.12), then for $|\eta|$ large enough,

$$
\left(\eta^{\mu}-\underline{A}\right) b(\eta)-1=r_{R}(\eta), \quad b(\eta)\left(\eta^{\mu}-\underline{A}\right)-1=r_{L}(\eta),
$$

with

$$
r_{R}(\eta) \in C_{G}^{-\infty}(\Sigma ; \gamma, \gamma, \varepsilon), \quad r_{L}(\eta) \in \mathcal{S}(\Sigma, \mathcal{L}(\mathcal{D}(\underline{A})))
$$

for suitable $\varepsilon>0$. In particular, $\left(\eta^{\mu}-\underline{A}\right): \mathcal{D}(\underline{A}) \rightarrow \mathcal{H}_{p}^{0, \gamma}(\mathbb{B})$ is invertible for sufficiently large $|\eta|$.

Proof. Since $A$ is a local operator, we can write $\left(\eta^{\mu}-\underline{A}\right)=\sigma\left(\eta^{\mu}-\underline{A}\right) \sigma_{0}+(1-\sigma)\left(\eta^{\mu}-A\right)\left(1-\sigma_{1}\right)$ with cut-off functions as above. Then

$$
\left(\eta^{\mu}-\underline{A}\right) b(\eta)=\sigma\left(\eta^{\mu}-\underline{A}\right) \sigma_{0} b(\eta)+(1-\sigma)\left(\eta^{\mu}-\underline{A}\right)\left(1-\sigma_{1}\right) b(\eta) .
$$

To treat the first summand choose a representation of $b(\eta)$ as in Lemma 3.9 with $\widetilde{\sigma} \sigma_{0}=\sigma_{0}$. Then

$$
\sigma\left(\eta^{\mu}-\underline{A}\right) \sigma_{0} b(\eta)=\sigma+\sigma\left(\eta^{\mu}-\underline{A}\right) \sigma_{0} r(\eta),
$$

where the second term on the right-hand side belongs to $C_{G}^{-\infty}(\Sigma ; \gamma, \gamma, \varepsilon)$, since $A$ maps $\mathcal{C}_{\mathcal{E}^{\prime}}^{\infty, \gamma}$ to $\mathcal{C}_{\text {comp }}^{\infty}$ (int $\left.\mathbb{B}\right)$. For the second summand we choose for $b(\eta)$ a representation with $\widetilde{\sigma}_{0}$ such that $\sigma_{1} \widetilde{\sigma}_{0}=\widetilde{\sigma}_{0}$. Then

$$
(1-\sigma)\left(\eta^{\mu}-\underline{A}\right)\left(1-\sigma_{1}\right) b(\eta) \equiv(1-\sigma)+(1-\sigma)\left(\left(\eta^{\mu}-A\right) p(\eta)-1\right)\left(1-\widetilde{\sigma}_{1}\right)
$$

modulo a remainder of the prescribed form. Since $p(\eta)$ is a parametrix to $\eta^{\mu}-\underline{A}$ in the interior of $\mathbb{B}$, the second term on the right-hand side is of the form $(1-\sigma) d(\eta)\left(1-\widetilde{\sigma}_{1}\right)$ with $d(\eta) \in L^{-\infty}(2 \mathbb{B} ; \Sigma)$, hence is a remainder of the desired form. The considerations for $b(\eta)\left(\eta^{\mu}-\underline{A}\right)$ are analogous.

To finish the proof of Theorem 3.5.b), it remains to modify $b(\eta)$ in such a way that we obtain the inverse of $\eta^{\mu}-\underline{A}$. To do so, we may assume that $r_{R}(\eta)$ of Proposition 3.10 satisfies $\left\|r_{R}(\eta)\right\|_{\mathcal{L}\left(\mathcal{H}_{p}^{0, \gamma}(\mathbb{B})\right)} \leq \frac{1}{2}$ for all $\eta \in \Sigma$ (otherwise we multiply $r_{R}(\eta)$ with a suitable zero excision function $\chi(\eta)$ ). Then $1+r_{R}(\eta)$ is invertible in $\left.\mathcal{H}_{p}^{0, \gamma}(\mathbb{B})\right)$ for all $\eta \in \Sigma$ and

$$
\left(1+r_{R}(\eta)\right)^{-1}=1-r_{R}(\eta)+r_{R}(\eta)\left(1+r_{R}(\eta)\right)^{-1} r_{R}(\eta)=: 1+s(\eta) .
$$

Clearly, $s(\eta)$ belongs to $C_{G}^{-\infty}(\Sigma ; \gamma, \gamma, \varepsilon)$, again. Hence, by Proposition 3.10 and (3.10),

$$
\left(\eta^{\mu}-\underline{A}\right)^{-1}=b(\eta)\left(1+r_{R}(\eta)\right)^{-1}=b(\eta)+b(\eta) s(\eta)=\widetilde{b}(\eta)+\widetilde{r}(\eta)
$$


for large $|\eta|$, with $\widetilde{r}(\eta) \in C_{G}^{-\mu}(\Sigma ; \gamma, \gamma, \varepsilon)$ (note that the smoothing Mellin term in 3.10) belongs to $\left.C_{M+G}^{-\mu}(\Sigma ; \gamma-\varepsilon, \gamma+\mu-\varepsilon, \mu) \subset C_{G}^{-\mu}(\Sigma ; \gamma, \gamma, \varepsilon)\right)$.

\section{Spectral invariance and bounded imaginary powers}

4.1. Independence on the choice of $1<p<\infty$. A closed extension $\underline{A}$ of $A$ in $\mathcal{H}_{p_{0}}^{0, \gamma}(\mathbb{B})$ with domain $\mathcal{D}\left(A_{\text {min }}\right) \oplus \underline{\mathcal{E}}$ as in Theorem 2.8 induces closed extensions $\underline{A}_{p}$ in $\mathcal{H}_{p}^{0, \gamma}(\mathbb{B})$ for all $1<p<\infty$ by letting $A$ act on

$$
\mathcal{D}\left(\underline{A}_{p}\right)=\mathcal{D}_{p}\left(A_{\min }\right) \oplus \underline{\mathcal{E}}
$$

where $\mathcal{D}_{p}\left(A_{\min }\right)$ is the domain of the closure of $A$ in $\mathcal{H}_{p}^{0, \gamma}(\mathbb{B})$.

THEOREM 4.1. Suppose $\underline{A}=\underline{A}_{p_{0}}$ satisfies the assumptions of Theorem 3.5 for one $p_{0}$, and let $c(\eta)$ be the inverse of $\eta^{\mu}-\underline{A}_{p_{0}}$ for $|\eta| \geq R$. Then $c(\eta),|\eta| \geq R$, also yields the inverse of $\eta^{\mu}-\underline{A}_{p}$ for all $1<p<\infty$.

Proof. By (3.13), $c(\eta)=b(\eta)\left(1+r_{R}(\eta)\right)^{-1}$ with $b(\eta)=b_{R}(\eta)+\sigma_{0} g(\eta) \sigma_{0}$. According to Proposition 3.6, $b_{R}(\eta)$ maps $\mathcal{H}_{p}^{0, \gamma}(\mathbb{B})$ into $\mathcal{D}_{p}\left(A_{\min }\right)$. By Proposition 3.8, $\sigma_{0} g(\eta) \sigma_{0}$ maps into $\mathcal{C}_{\underline{\mathcal{E}}^{\prime}}^{\infty, \gamma}(\mathbb{B})$, which is a subspace of $\mathcal{D}\left(\underline{A}_{p}\right)$. Hence

$$
c(\eta): \mathcal{H}_{p}^{0, \gamma}(\mathbb{B}) \longrightarrow \mathcal{D}(\underline{A})=\mathcal{D}\left(\underline{A}_{p}\right)
$$

for all $1<p<\infty$. Moreover, $c(\eta)\left(\eta^{\mu}-\underline{A}_{p}\right)=1$ on $\mathcal{C}_{\text {comp }}^{\infty}($ int $\mathbb{B}) \oplus \underline{\mathcal{E}}$ for each $p$, since this is true for $p=p_{0}$ and the left-hand side of the latter equation is independent of $p$ on $\mathcal{C}_{\text {comp }}^{\infty}($ int $\mathbb{B}) \oplus \underline{\mathcal{E}}$. Similarly, $\left(\eta^{\mu}-\underline{A}_{p}\right) c(\eta)=1$ on $\mathcal{C}_{\text {comp }}^{\infty}($ int $\mathbb{B})$ for all $p$. Thus a density argument gives the result.

Equation (3.8) together with Proposition 3.8 shows that $\left(\eta^{\mu}-\underline{\widehat{A}}_{p}\right)^{-1}$ is the sum of two principal edge symbols, namely those of an operator in $C_{O}^{-\mu}(\Sigma)$ and one in $R_{G}^{-\mu}(\Sigma ; \gamma, \gamma, \varepsilon)$. Arguing similarly as above, we therefore obtain

THEOREM 4.2. If $\underline{A}=\underline{A}_{p}$ satisfies ellipticity assumptions (E1), (E2), and (E3) of Section 3.2 for one $p$, then automatically for all $1<p<\infty$.

4.2. Bounded imaginary powers. In the paper [3] we have shown that the closure of a cone differential operator - under ellipticity conditions (E1) and (E3) with $\underline{\mathcal{E}}=\{0\}$ - posseses bounded imaginary powers whose operator-norm in $\mathcal{H}_{p}^{0, \gamma}(\mathbb{B})$ can be estimated by $c_{p} e^{\theta|z|}$, where $\theta$ is the angle determining $\Lambda=\Lambda_{\theta}$. We also had pointed out in Remark 5.5 of [3] that the validity of this result 'only' relies on the structure of the resolvent and not on the fact that we dealt with the minimal extension. Theorem 3.5 now states that the resolvent of a general closed extension has exactly this required structure (in [3] we described the resolvent $(\lambda-\bar{A})^{-1}$ in terms of anisotropic symbols, while here we described $\left(\eta^{\mu}-\underline{A}\right)^{-1}$; both ways, however, are obviously equivalent). Thus we have the following result:

THEOREM 4.3. Let $\underline{A}$ be a closed extension of a cone differential operator A, satisfying the ellipticity assumptions (E1), (E2), and (E3) with respect to $\Lambda=\Lambda_{\theta}$. Then the resolvent $(\lambda-\underline{A})^{-1}$ exists for large $\lambda$ in $\Lambda$, and its norm in $\mathcal{H}_{p}^{0, \gamma}(\mathbb{B})$ decays like $1 /|\lambda|$. Moreover, there exists a constant $c \geq 0$ such that $\underline{A}+c$ has bounded imaginary powers and, for a certain constant $c_{p} \geq 0$,

$$
\left\|(\underline{A}+c)^{i \varrho}\right\|_{\mathcal{L}\left(\mathcal{H}_{p}^{0, \gamma}(\mathbb{B})\right)} \leq c_{p} e^{\theta|\varrho|} \quad \forall \varrho \in \mathbb{R} .
$$

As the construction of complex powers shows, we can take $c=0$ if $\underline{A}$ has no spectrum in $\Lambda_{\theta}$.

Let us mention that the operator $A+c$ does not satisfy the assumption of constant coefficients near the boundary (since we have to write $c=t^{-\mu}\left(t^{\mu} c\right)$ ). However, the structure of the resolvent remains uneffected by the shift with a constant $c$. 


\section{The Laplace-Beltrami operator}

Let the interior of $\mathbb{B}$ be equipped with a metric that coincides with $d t^{2}+t^{2} g$ on $] 0,1[\times \partial \mathbb{B}$ for some fixed metric $g$ on $\partial \mathbb{B}$ (straight conical degeneracy). The associated Laplacian $\Delta$ is a second order cone differential operator, and

$$
\Delta=t^{-2}\left\{\left(t \partial_{t}\right)^{2}+(n-1) t \partial_{t}+\Delta_{\partial}\right\}, \quad n=\operatorname{dim} \partial \mathbb{B},
$$

near the boundary of $\mathbb{B}$, where $\Delta_{\partial}$ denotes the Laplacian on $\partial \mathbb{B}$ with respect to $g$.

Clearly, $-\Delta$ satisfies ellipticity condition (E1) of Section 3.2 for any sector $\Lambda$ not containing positive reals.

5.1. The conormal symbol. Let us first analyze the inverse of the conormal symbol

$$
\sigma_{M}^{2}(\Delta)(z)=z^{2}-(n-1) z+\Delta_{\partial} .
$$

To this end denote by $0=\lambda_{0}>\lambda_{1}>\ldots$ the eigenvalues of $\Delta_{\partial}$ and by $E_{0}, E_{1}, \ldots$ the corresponding eigenspaces. Moreover, let $\pi_{j} \in \mathcal{L}\left(L_{2}(\partial \mathbb{B})\right)$ be the orthogonal projection onto $E_{j}$.

The non-bijectivity points of $\sigma_{M}^{2}(\Delta)$ are exactly the points $z=q_{j}^{+}$and $z=q_{j}^{-}$with

$$
q_{j}^{ \pm}=\frac{n-1}{2} \pm \sqrt{\left(\frac{n-1}{2}\right)^{2}-\lambda_{j}}, \quad j \in \mathbb{N}_{0}
$$

Note the symmetry $q_{j}^{+}=(n-1)-q_{j}^{-}$. It is straightforward to calculate that

$$
\left(z^{2}-(n-1) z+\Delta_{\partial}\right)^{-1}=\sum_{j=0}^{\infty} \frac{1}{q_{j}^{+}-q_{j}^{-}}\left(\frac{1}{z-q_{j}^{+}}-\frac{1}{z-q_{j}^{-}}\right) \pi_{j} .
$$

Hence, in case $\operatorname{dim} \mathbb{B} \geq 3$,

$$
\left(z^{2}-(n-1) z+\Delta_{\partial}\right)^{-1} \equiv \pm \frac{1}{q_{j}^{+}-q_{j}^{-}} \pi_{j}\left(z-q_{j}^{ \pm}\right)^{-1} \quad \text { near } z=q_{j}^{ \pm}
$$

modulo holomorphic germs. In case $\operatorname{dim} \mathbb{B}=2$ the same formula holds near $z=q_{j}^{ \pm}$if $j \geq 1$ but, since then $q_{0}^{+}=q_{0}^{-}=0$,

$$
\left(z^{2}+\Delta_{\partial}\right)^{-1} \equiv \pi_{0} z^{-2} \quad \text { near } z=0 .
$$

For $\operatorname{dim} \mathbb{B}=1$, this simplifies to $\sigma_{M}^{2}(\Delta)^{-1}(z)=\left(z^{2}+z\right)^{-1}=\frac{1}{z}-\frac{1}{z+1}$ with only two poles in $q_{0}^{-}=-1$ and $q_{0}^{+}=0$ and associated 'eigenspace' $E_{0}=\mathbb{C}$.

5.2. Maximal domain and dilation invariance. With $q_{j}^{ \pm}$we associate the function space

$$
\mathcal{E}_{q_{j}^{ \pm}}=E_{j} \otimes \omega t^{-q_{j}^{ \pm}}=\left\{e(x) \omega(t) t^{-q_{j}^{ \pm}} \mid e \in E_{j}\right\}, \quad j \in \mathbb{N},
$$

and for $q_{0}^{ \pm}$we set

$$
\mathcal{E}_{q_{0}^{ \pm}}=\left\{\begin{array}{ll}
E_{0} \otimes \omega+E_{0} \otimes \omega \log t & \operatorname{dim} \mathbb{B}=2 \\
E_{0} \otimes \omega t^{q_{0}^{ \pm}} & \operatorname{dim} \mathbb{B} \neq 2
\end{array} .\right.
$$

Furthermore, for $\gamma \in \mathbb{R}$, set

$$
\left.I_{\gamma}=\left\{q_{j}^{ \pm} \mid j \in \mathbb{N}_{0}\right\} \cap\right] \frac{n+1}{2}-\gamma-2, \frac{n+1}{2}-\gamma\left[=\left\{q_{j}^{ \pm} \mid j \in \mathbb{N}_{0}\right\} \cap\right] \frac{n-1}{2}-\gamma-1, \frac{n-1}{2}-\gamma+1[.
$$

Applying Theorems 2.3 and 2.8 to $A=\Delta$, we get the following:

Proposition 5.1. The domain of the maximal extension of $\Delta$ in $\mathcal{H}_{p}^{0, \gamma}(\mathbb{B})$ is

$$
\mathcal{D}\left(\Delta_{\text {max }}\right)=\mathcal{D}\left(\Delta_{\text {min }}\right) \oplus \underset{q \in I_{\gamma}}{\oplus} \mathcal{E}_{q} .
$$

In case $q_{j}^{ \pm} \neq \frac{n+1}{2}-\gamma-2$ for all $j$, the minimal domain is $\mathcal{D}\left(\Delta_{\text {min }}\right)=\mathcal{H}_{p}^{2, \gamma+2}(\mathbb{B})$. 
Let us now describe the closed extensions $\Delta$ of $\Delta$ satisfying condition (E2) of Section 3.2. For convenience we shall call such extensions dilation invariant. A straightforward calculation (or an application of Lemmas 5.11 and 5.12 of $[\mathbf{1 0}$ ) yields:

Proposition 5.2. Consider $\Delta$ as an unbounded operator in $\mathcal{H}_{p}^{0, \gamma}(\mathbb{B})$. The dilation invariant extensions $\triangleq$ are precisely those with a domain of the form

$$
\mathcal{D}(\underline{\Delta})=\mathcal{D}\left(\Delta_{\min }\right) \oplus \underset{q \in I_{\gamma}}{\oplus} \underline{\mathcal{E}}_{q}, \quad \underline{\mathcal{E}}_{q} \text { subspace of } \mathcal{E}_{q},
$$

where in case $\operatorname{dim} \mathbb{B}=2$ either $\underline{\mathcal{E}}_{0}=\{0\}$ or $\underline{\mathcal{E}}_{0}=E_{0} \otimes \omega$ or $\underline{\mathcal{E}}_{0}=\mathcal{E}_{0}$, cf. (5.2).

Let us point out that in $(5.3)$ the sum is taken over all $q \in I_{\gamma}$ and that the summand $\underline{\mathcal{E}}_{q}=\{0\}$ may occur several times.

5.3. Adjoint operators. Since the scalar-product $\langle\cdot, \cdot\rangle_{0,0}$ of $\mathcal{H}_{2}^{0,0}(\mathbb{B})$ yields an identification of the dual space of $\mathcal{H}_{p}^{0, \gamma}(\mathbb{B})$ with $\mathcal{H}_{p^{\prime}}^{0,-\gamma}(\mathbb{B})$, the adjoint $\underline{\Delta}^{*}$ of an extension $\underline{\Delta}$ in $\mathcal{H}_{p}^{0, \gamma}(\mathbb{B})$ is the an unbounded operator in $\mathcal{H}_{p^{\prime}}^{0,-\gamma}(\mathbb{B})$ given by the action of $\Delta$ on the domain

$$
\mathcal{D}\left(\underline{\Delta}^{*}\right)=\left\{v \in \mathcal{H}_{p^{\prime}}^{0,-\gamma}(\mathbb{B}) \mid \exists f \in \mathcal{H}_{p^{\prime}}^{0,-\gamma}(\mathbb{B}) \forall u \in \mathcal{D}(\underline{\Delta}): \quad\langle v, \Delta u\rangle_{0,0}=\langle f, u\rangle_{0,0}\right\} .
$$

It is easy to see that $\Delta_{\min }^{*}=\Delta_{\max }$ and $\Delta_{\max }^{*}=\Delta_{\min }$.

We shall now compute explicitly the adjoints of the dilation invariant extensions. For an analysis of adjoints of general cone differential operators (in case $p=2$ ) see the paper $[\mathbf{1 0}$. Define

$$
[\cdot, \cdot]: \mathcal{D}_{p}^{\gamma}\left(\Delta_{\max }\right) \times \mathcal{D}_{p^{\prime}}^{-\gamma}\left(\Delta_{\max }\right) \longrightarrow \mathbb{C}, \quad[u, v]=\langle\Delta u, v\rangle_{0,0}-\langle u, \Delta v\rangle_{0,0},
$$

where the indices $\sigma, r$ in $\mathcal{D}_{r}^{\sigma}$ now indicate that we consider the Laplacian in the Sobolev space $\mathcal{H}_{r}^{0, \sigma}(\mathbb{B})$. Then the domain of the adjoint operator $\underline{\Delta}^{*}$ is just the orthogonal space (with respect to this pairing) to the domain of $\underline{\Delta}$, i.e.

$$
\mathcal{D}_{p^{\prime}}^{-\gamma}\left(\underline{\Delta}^{*}\right)=\mathcal{D}_{p}^{\gamma}(\underline{\Delta})^{\perp}
$$

Since $[u, v]=0$ whenever $u$ or $v$ belong to the minimal domain, we classify first which elements of $\oplus_{q \in I_{-\gamma}} \mathcal{E}_{q}$ are orthogonal to a given element of $\oplus_{q \in I_{\gamma}} \mathcal{E}_{q}$.

Let $u=e \omega t^{-q}$ with $q=q_{j}^{+}$or $q=q_{j}^{-}$and $e \in E_{j}$ for some fixed $j \in \mathbb{N}_{0}$. If $v_{ \pm}=f \omega t^{-q_{k}^{ \pm}}$with $f \in E_{k}$, an elementary calculation yields

$$
(\Delta u) \overline{v_{ \pm}}-u \overline{\Delta v_{ \pm}}=2\left(q_{k}^{ \pm}-q\right) e \bar{f} \omega \omega^{\prime} t^{-q-q_{k}^{ \pm}-1}
$$

hence $\left[u, v_{ \pm}\right]=0$ if and only if $q_{k}^{ \pm}=q$ or $\langle e, f\rangle_{L_{2}(\partial \mathbb{B})}=0$.

If $\operatorname{dim} \mathbb{B}=2$ and $u=c \omega+d \omega \log t$ with $c, d \in \mathbb{C}$ and $v_{ \pm}=f \omega t^{-q_{k}^{ \pm}}$with $f \in E_{k}$ and $k \neq 0$ then

$$
(\Delta u) \overline{v_{ \pm}}-u \overline{\Delta v_{ \pm}}=2 \bar{f}\left(d+c q_{k}^{ \pm}+d q_{k}^{ \pm} \log t\right) \omega \omega^{\prime} t^{-q_{k}^{ \pm}-1}
$$

hence $\left[u, v_{ \pm}\right]=0$, since $\langle 1, f\rangle_{L_{2}(\partial \mathbb{B})}=0$. If $u$ is as before and $v=c_{0} \omega+d_{0} \omega \log t$ with $c_{0}, d_{0} \in \mathbb{C}$, then

$$
(\Delta u) \bar{v}-u \overline{\Delta v}=2\left(\bar{c}_{0} d-\bar{d}_{0} c\right) \omega \omega^{\prime} t^{-3},
$$

hence if both $u$ and $v$ are different from zero, $[u, v]=0$ if and only if $\bar{v}$ is a multiple of $u$.

From this we derive the following description of adjoints of dilation invariant extensions:

THEOREM 5.3. Let $\triangleq$ be a dilation invariant extension of $\Delta$ in $\mathcal{H}_{p}^{0, \gamma}(\mathbb{B})$ with domain

$$
\mathcal{D}_{p}^{\gamma}(\underline{\Delta})=\mathcal{D}_{p}^{\gamma}\left(\Delta_{\min }\right) \oplus \underset{q \in I_{\gamma}}{\oplus} \underline{\mathcal{E}}_{q}
$$

as described in Proposition 5.2. Then the domain of the adjoint $\triangleq^{*}$ is

$$
\mathcal{D}_{p^{\prime}}^{-\gamma}\left(\underline{\Delta}^{*}\right)=\mathcal{D}_{p^{\prime}}^{-\gamma}\left(\Delta_{\min }\right) \oplus \underset{q \in I_{\gamma}}{\oplus} \underline{\mathcal{E}}_{q}^{\perp}
$$


where the spaces $\underline{\mathcal{E}}_{q_{j}^{ \pm}}^{\perp}$ are defined as follows:

i) If either $q_{j}^{ \pm} \neq 0$ or $\operatorname{dim} \mathbb{B} \neq 2$, there exists a unique subspace $\underline{E}_{j} \subset E_{j}$ such that $\underline{\mathcal{E}}_{q_{j}^{ \pm}}=$ $\underline{E}_{j} \otimes \omega t^{-q_{j}^{ \pm}}$. Then we set

$$
\underline{\mathcal{E}}_{q_{j}^{ \pm}}^{\perp}=\underline{E}_{j}^{\perp} \otimes \omega t^{-q_{j}^{\mp}}
$$

where $\underline{E}_{j}^{\perp}$ is the orthogonal complement of $\underline{E}_{j}$ in $E_{j}$ with respect to the $L_{2}(\partial \mathbb{B})$-scalar product.

ii) If $\operatorname{dim} \mathbb{B}=2$ and $q_{j}^{ \pm}=0$ define $\underline{\mathcal{E}}_{0}^{\perp}=\{0\}$ if $\underline{\mathcal{E}}_{0}=\mathcal{E}_{0}, \underline{\mathcal{E}}_{0}^{\perp}=\mathcal{E}_{0}$ if $\underline{\mathcal{E}}_{0}=\{0\}$, and $\underline{\mathcal{E}}_{0}^{\perp}=\underline{\mathcal{E}}_{0}$ if $\underline{\mathcal{E}}_{0}=E_{0} \otimes \omega$.

Note that $\underline{\mathcal{E}}_{q_{j}^{ \pm}}^{\perp}$ is a subspace of $\mathcal{E}_{q_{j}^{\mp}}$ or, equivalently, $\mathcal{E}_{q}^{\perp}$ is a subspace of $\mathcal{E}_{(n-1)-q}$.

COROLlary 5.4. The selfadjoint dilation invariant extensions $\Delta$ of $\Delta$ in $\mathcal{H}_{2}^{0,0}(\mathbb{B})$ are those with a domain of the form

$$
\mathcal{D}_{2}^{0}(\underline{\Delta})=\mathcal{D}_{2}^{0}\left(\Delta_{\text {min }}\right) \oplus \underset{q \in I_{0}}{\oplus} \underline{\mathcal{E}}_{q}
$$

with $\underline{\mathcal{E}}_{q}^{\perp}=\underline{\mathcal{E}}_{(n-1)-q}$ for all $q \in I_{0}$ (in particular $\underline{\mathcal{E}}_{0}=E_{0} \otimes \omega$ in case $\operatorname{dim} \mathbb{B}=2$ ).

Applying Theorems 8.3 and 8.12 of [10], the Friedrichs extension of $\Delta$ has the domain

$$
\mathcal{D}_{2}^{0}(\underline{\Delta})=\left\{\begin{array}{ll}
\mathcal{D}_{2}^{0}\left(\Delta_{\text {min }}\right) \oplus \underset{\substack{q \in I_{0} \\
\operatorname{Re} q<0}}{\oplus} \mathcal{E}_{q} \oplus\left(E_{0} \otimes \omega\right) & \operatorname{dim} \mathbb{B}=2 \\
\mathcal{D}_{2}^{0}\left(\Delta_{\text {min }}\right) \oplus \underset{\substack{q \in I_{0} \\
\operatorname{Re} q \leq \frac{n-1}{2}}}{\bigoplus} \mathcal{E}_{q} & \operatorname{dim} \mathbb{B} \neq 2
\end{array} .\right.
$$

In particular, the Friedrichs extension is dilation invariant.

REMARK 5.5. All the results of Sections 5.2 and 5.3 hold true in an analogous form for the model cone operator $\widehat{\Delta}$ considered as an unbounded operator in $\mathcal{K}_{p}^{0, \gamma}\left(\partial \mathbb{B}^{\wedge}\right)$.

5.4. Elliptic extensions. Proposition 5.2 provides a complete description of the closed extensions $\underline{\Delta}$ of $\Delta$ such that $-\underline{\Delta}$ satisfies the ellipticity conditions (E1) and (E2) of Section 3.2. We shall now discuss condition (E3), assuming that $|\gamma|<\frac{1}{2} \operatorname{dim} \mathbb{B}=\frac{n+1}{2}$ (the choice of this range is connected to the scale of natural $L_{p}$-spaces on $\mathbb{B}$ as we shall explain below). It turns out that for each given $\gamma$ we find at least one extension having property (E3); in case $\operatorname{dim} \mathbb{B} \leq 3$ we find more than one. However, the extensions we describe might not represent all possible choices.

TheOREM 5.6. Let $\Lambda \subset \mathbb{C} \backslash \mathbb{R}_{+}$be an arbitrary sector. Consider $-\Delta$ as an unbounded operator in $\mathcal{H}_{p}^{0, \gamma}(\mathbb{B})$ and assume $\operatorname{dim} \mathbb{B} \geq 4$. Then conditions (E1), (E2), and (E3) of Section 3.2 are fulfilled by $-\Delta_{\max }$ in case $0 \leq \gamma<\frac{1}{2} \operatorname{dim} \mathbb{B}$ and by $-\Delta_{\min }$ in case $-\frac{1}{2} \operatorname{dim} \mathbb{B}<\gamma \leq 0$.

The assumption on the dimension of $\mathbb{B}$ in the previous theorem ensures that $\Delta$ in $\mathcal{H}_{2}^{0,0}(\mathbb{B})$ is essentially self-adjoint or, in other words, the inverted conormal symbol has no pole in the interval $I_{0}$. We shall omit the proof of this theorem, since it is a simpler version of that for the following one (cf. also the proof of Theorem 7.1 in [3]).

Theorem 5.7. Consider $-\Delta$ as an unbounded operator in $\mathcal{H}_{p}^{0, \gamma}(\mathbb{B})$, assume $\operatorname{dim} \mathbb{B} \leq 3$, and let $|\gamma|<\frac{1}{2} \operatorname{dim} \mathbb{B}$. An extension $-\underline{\Delta}$ satisfies conditions (E1), (E2), and (E3) of Section 3.2 for any sector $\Lambda \subset \mathbb{C} \backslash \mathbb{R}_{+}$, provided we choose its domain

$$
\mathcal{D}_{p}^{\gamma}(\underline{\Delta})=\mathcal{D}_{p}^{\gamma}\left(\Delta_{\min }\right) \oplus \underset{q \in I_{\gamma}}{\oplus} \underline{\mathcal{E}}_{q}
$$

according to the following rules: 
(i) If $q \in I_{\gamma} \cap I_{-\gamma}$, then $\underline{\mathcal{E}}_{q}^{\perp}=\underline{\mathcal{E}}_{(n-1)-q}$.

(ii) If $\gamma \geq 0$ and $q \in I_{\gamma} \backslash I_{-\gamma}$, then $\underline{\mathcal{E}}_{q}=\mathcal{E}_{q}$.

(iii) If $\gamma \leq 0$ and $q \in I_{-\gamma} \backslash I_{\gamma}$, then $\underline{\mathcal{E}}_{q}=\{0\}$.

In particular, $\mathcal{D}_{p}^{\gamma}(\underline{\Delta})=\mathcal{D}_{p}^{\gamma}\left(\Delta_{\max }\right)$ if $\gamma \geq 1$ and $\mathcal{D}_{p}^{\gamma}(\underline{\Delta})=\mathcal{D}_{p}^{\gamma}\left(\Delta_{\min }\right)$ if $\gamma \leq-1$.

Proof. By Theorem 4.2 we may assume that $p=2$, and by duality it suffices to treat the case $\gamma \geq 0$. Let $\underline{\Delta}_{0}$ denote the selfadjoint extension of $\Delta$ in $\mathcal{H}_{p}^{0,0}(\mathbb{B})$ with $\mathcal{D}_{2}^{\gamma}(\underline{\Delta}) \subset \mathcal{D}_{2}^{0}\left(\underline{\Delta}_{0}\right)$. Such an extension always exists due to assumption (i) on the domain of $\underline{\Delta}$ and by Corollary 5.4; its domain is

$$
\mathcal{D}_{2}^{0}\left(\underline{\Delta}_{0}\right)=\mathcal{D}_{2}^{0}\left(\Delta_{\text {min }}\right) \oplus \underset{q \in I_{0} \backslash I_{-\gamma}}{\oplus} \mathcal{E}_{q} \oplus \underset{q \in I_{\gamma} \cap I_{-\gamma}}{\oplus} \underline{\mathcal{E}}_{q} .
$$

If we then pass to the associated model cone operators and use Remark 5.5, we get that

$$
\lambda+\underline{\widehat{\Delta}}: \mathcal{D}_{2}^{\gamma}(\underline{\widehat{\Delta}}) \longrightarrow \mathcal{K}_{2}^{0, \gamma}\left(\partial \mathbb{B}^{\wedge}\right), \quad \lambda \notin \overline{\mathbb{R}}_{+},
$$

is injective, since $\operatorname{spec}\left(-\widehat{\widehat{\Delta}}_{0}\right) \subset \overline{\mathbb{R}}_{+}$and $\mathcal{D}_{2}^{\gamma}(\underline{\widehat{\Delta}}) \subset \mathcal{D}_{2}^{0}\left(\widehat{\widehat{\Delta}}_{0}\right)$.

By Theorem 5.3 (in the formulation for model cone operators), the adjoint $\widehat{\widehat{\Delta}}^{*}$ of $\widehat{\widehat{\Delta}}$ has the domain

$$
\mathcal{D}_{2}^{-\gamma}\left(\underline{\widehat{\Delta}}^{*}\right)=\mathcal{D}_{2}^{-\gamma}\left(\widehat{\Delta}_{\min }\right) \oplus \underset{q \in I_{\gamma} \cap I_{-\gamma}}{\oplus} \underline{\mathcal{E}}_{q} .
$$

Now let $\lambda \in \mathbb{C} \backslash \overline{\mathbb{R}}_{+}$and $u \in \mathcal{D}_{2}^{-\gamma}\left(\underline{\widehat{\Delta}}^{*}\right)$ be an element of the kernel of $\lambda+\underline{\widehat{\Delta}}^{*}$, i.e. $(\lambda+\widehat{\Delta}) u=0$. We shall show now that this implies $u=0$. To this end write $u=u_{0}+u_{1}$ with $u_{0} \in \mathcal{D}_{2}^{-\gamma}\left(\widehat{\Delta}_{\text {min }}\right)$ and $u_{1} \in \underset{q \in I_{\gamma} \cap I_{-\gamma}}{\oplus} \underline{\mathcal{E}}_{q}$. Note that $u_{0}, u_{1} \in \mathcal{K}_{2}^{0,0}\left(\partial \mathbb{B}^{\wedge}\right)$ by the assumption on the dimension of $\mathbb{B}$ and the structure of the domain of $\underline{\Delta}$. Since $\widehat{\Delta} u_{1} \in \mathcal{C}_{\mathrm{comp}}^{\infty}(\operatorname{int} \mathbb{B}$ ) (as this is true for any linear combination of functions from the spaces $\left.\mathcal{E}_{q}\right)$, we obtain $(\lambda+\widehat{\Delta}) u_{0}=-\lambda u_{1}-\widehat{\Delta} u_{1} \in \mathcal{K}_{2}^{0,0}\left(\partial \mathbb{B}^{\wedge}\right)$. But this means that

$$
u_{0} \in \mathcal{D}_{2}^{0}\left(\widehat{\Delta}_{\max }\right) \cap \mathcal{D}_{2}^{-\gamma}\left(\widehat{\Delta}_{\min }\right)=\mathcal{D}_{2}^{0}\left(\widehat{\Delta}_{\min }\right) \oplus \underset{q \in I_{0} \backslash I_{-\gamma}}{\oplus} \mathcal{E}_{q} \subset \mathcal{D}_{2}^{0}\left(\widehat{\widehat{\Delta}}_{0}\right) .
$$

The last inclusion is valid by construction of $\underline{\Delta}_{0}$. This yields $u \in \mathcal{D}_{2}^{0}\left(\widehat{\Delta}_{0}\right)$ and $(\lambda+\widehat{\Delta}) u=0$, hence $u=0$, since $\operatorname{spec}\left(-\widehat{\widehat{\Delta}}_{0}\right) \subset \overline{\mathbb{R}}_{+}$.

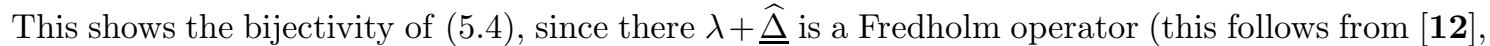
Proposition 1.3.16, together with a parametrix construction on $\partial \mathbb{B}^{\wedge}$ as in the proof of Proposition 2.11), hence has closed range.

5.5. The Cauchy Problem. Let $1<p<\infty$ and let $L_{p}(\mathbb{B})$ denote the $L_{p}$-space on int $\mathbb{B}$ associated to the measure induced by the conical metric on int $\mathbb{B}$. Then

$$
L_{p}(\mathbb{B})=\mathcal{H}_{p}^{0, \gamma_{p}}(\mathbb{B}), \quad \gamma_{p}=(n+1)\left(\frac{1}{2}-\frac{1}{p}\right) .
$$

In fact, away from the boundary these spaces coincide by definition; thus it suffices to consider functions supported close to the boundary. But then, cf. Definition 2.2,

$$
\|u\|_{\mathcal{H}_{p}^{0, \gamma_{p}(\mathbb{B})}}^{p}=\int_{[0,1] \times \partial \mathbb{B}}\left|t^{\frac{n+1}{2}-\gamma_{p}} u(t, x)\right|^{p} \frac{d t}{t} d x=\int_{[0,1] \times \partial \mathbb{B}}|u(t, x)|^{p} t^{n} d t d x=\|u\|_{L_{p}(\mathbb{B})}^{p} .
$$

Clearly, $\left|\gamma_{p}\right|<\frac{n+1}{2}=\frac{1}{2} \operatorname{dim} \mathbb{B}$ when $p$ ranges from 1 to $\infty$. Therefore the results of the previous Section 5.4 can be applied to the Laplacian in $L_{p}(\mathbb{B}), 1<p<\infty$.

Combining these results with Theorem 4.3 and the Dore-Venni theorem (Theorem 3.2 in [5), one obtains maximal regularity for solutions of the Cauchy problem: 
THE RESOLVENT OF CLOSED EXTENSIONS OF CONE DIFFERENTIAL OPERATORS

TheOREM 5.8. Consider $\Delta$ as an unbounded operator in $L_{p}(\mathbb{B}), 1<p<\infty$. For each closed extension $\Delta$ from Theorems 5.6 or 5.7 associated with $\gamma=\gamma_{p}$, the Cauchy problem

$$
\dot{u}(t)-\Delta u(t)=f(t) \quad \text { on } \quad 0<t<T, \quad u(0)=0,
$$

has for any $f \in L_{q}\left([0, T], L_{p}(\mathbb{B})\right), 1<q<\infty$, a unique solution

$$
u \in W_{q}^{1}\left([0, T], L_{p}(\mathbb{B})\right) \cap L_{q}\left([0, T], \mathcal{D}_{p}^{\gamma_{p}}(\underline{\Delta})\right) .
$$

\section{Appendix: Parameter-dependent cone pseudodifferential operators}

We try to give a concise review of the calculus of parameter-dependent pseudodifferential operators on $\mathbb{B}$ introduced by Schulze [21], [22]. Our presentation follows [26] and [11]. While there the parameter-space was $\mathbb{R}^{q}$, we focus here on a subsector of the complex plane. The proofs pass over to this situation without any changes, and thus will be dropped here.

We split the presentation into two parts: In Sections 6.1 to 6.3 we describe a sub-calculus of flat operators. Under suitable ellipticity assumptions it already allows the construction of a rough parametrix to $\eta^{\mu}-A$ for a $\mu$-th order cone differential operator $A$. To describe the resolvent $\left(\eta^{\mu}-A\right)^{-1}$ we need to enlarge this calculus. This shall be explained starting with Section 6.4.

In the following, $\Sigma$ is a closed sector in the complex plane (identified with $\mathbb{R}^{2}$ ) containing zero, i.e.

$$
\Sigma=\left\{\eta \in \mathbb{C} \mid \theta_{1} \leq \arg \eta \leq \theta_{2}\right\} \cup\{0\}, \quad-\pi \leq \theta_{1}, \theta_{2} \leq \pi .
$$

For a Fréchet space $E$, we let $\mathcal{C}^{\infty}(\Sigma, E)$ denote the space of all continuous functions $\Sigma \rightarrow E$ that are smooth in the interior of $\Sigma$ and whose derivatives have continuous extensions to the whole sector $\Sigma$. A subspace is $\mathcal{S}(\Sigma, E)$, consisting of those functions that decay rapidly in $\eta$ as $|\eta| \rightarrow \infty$ in $\Sigma$.

We fix a smooth positive function [.] with $[\eta]=|\eta|$ if $|\eta| \geq 1$. Also recall that a cut-off function is a non-negative decreasing function in $\mathcal{C}_{\text {comp }}^{\infty}([0,1[)$ which is identically 1 near zero.

6.1. Smoothing elements of the flat calculus. The space $\mathcal{C}^{\infty, \infty}(\mathbb{B})$, consisting of all functions that are smooth in the interior of $\mathbb{B}$ and vanish to infinite order at the boundary, is Fréchet in a natural way. Taking the projective tensor product yields the space

$$
\mathcal{C}^{\infty, \infty}(\mathbb{B} \times \mathbb{B})=\mathcal{C}^{\infty, \infty}(\mathbb{B}) \widehat{\otimes}_{\pi} \mathcal{C}^{\infty, \infty}(\mathbb{B}) .
$$

Definition 6.1. Let $C_{G}^{-\infty}(\Sigma)_{\infty}$ be the space of all operator-families $r(\eta): \mathcal{C}^{\infty, \infty}(\mathbb{B}) \rightarrow \mathcal{C}^{\infty, \infty}(\mathbb{B})$, $\eta \in \Sigma$, such that

$$
(r(\eta) u)(y)=\int_{\mathbb{B}} k_{r}\left(\eta, y, y^{\prime}\right) u\left(y^{\prime}\right) d y^{\prime},
$$

where $d y^{\prime}$ is a measure induced by a conic metric on $\mathbb{B}$ and the kernel $k_{r} \in \mathcal{S}\left(\Sigma, \mathcal{C}^{\infty, \infty}(\mathbb{B} \times \mathbb{B})\right)$ is rapidly decreasing in $\eta \in \Sigma$.

Besides this kind of smoothing operators - which act globally on $\mathbb{B}$ and decay rapidly in the parameter - we shall also need a class of smoothing operators that are localized near the boundary but have a non-trivial dependence on $\eta \in \Sigma$.

To this end let $\mathcal{S}^{\infty}\left(\partial \mathbb{B}^{\wedge}\right)$ denote the space of smooth functions $\mathbb{R}_{+} \times \partial \mathbb{B} \rightarrow \mathbb{C}$ that vanish to infinite order in $t=0$ and decrease rapidly for $t \rightarrow \infty$. We then define

$$
\mathcal{S}^{\infty}\left(\partial \mathbb{B}^{\wedge} \times \partial \mathbb{B}^{\wedge}\right)=\mathcal{S}^{\infty}\left(\partial \mathbb{B}^{\wedge}\right) \widehat{\otimes}_{\pi} \mathcal{S}^{\infty}\left(\partial \mathbb{B}^{\wedge}\right) .
$$

Definition 6.2. Let $R_{G}^{\mu}(\Sigma)_{\infty}, \mu \in \mathbb{R}$, denote the space of all operator-families $a(\eta): \mathcal{S}^{\infty}\left(\partial \mathbb{B}^{\wedge}\right) \rightarrow$ $\mathcal{S}^{\infty}\left(\partial \mathbb{B}^{\wedge}\right), \eta \in \Sigma$, such that

$$
(a(\eta) u)(t, x)=[\eta]^{n+1} \int_{\partial \mathbb{B}^{\wedge}} k_{a}\left(\eta, t[\eta], x, t^{\prime}[\eta], x^{\prime}\right) u\left(t^{\prime}, x^{\prime}\right) t^{\prime n} d t^{\prime} d x^{\prime},
$$


with an integral kernel satisfying

$$
k_{a}\left(\eta, t, x, t^{\prime}, x^{\prime}\right) \in S_{c l}^{\mu}\left(\Sigma, \mathcal{S}^{\infty}\left(\partial \mathbb{B}^{\wedge} \times \partial \mathbb{B}^{\wedge}\right)\right):=S_{c l}^{\mu}(\Sigma) \widehat{\otimes}_{\pi} \mathcal{S}^{\infty}\left(\partial \mathbb{B}^{\wedge} \times \partial \mathbb{B}^{\wedge}\right) .
$$

Using such operator-families, the so-called flat Green symbols are defined as follows:

Definition 6.3. For $\mu \in \mathbb{R}$ let $C_{G}^{\mu}(\Sigma)_{\infty}$ denote the space of all operator-families $g(\eta): \mathcal{C}^{\infty, \infty}(\mathbb{B}) \rightarrow$ $\mathcal{C}^{\infty, \infty}(\mathbb{B}), \eta \in \Sigma$, such that

$$
g(\eta)=\sigma a(\eta) \sigma_{0}+r(\eta)
$$

for some cut-off functions $\sigma, \sigma_{0} \in \mathcal{C}^{\infty}\left(\left[0,1[), a \in R_{G}^{\mu}(\Sigma)_{\infty}\right.\right.$, and $r \in C_{G}^{-\infty}(\Sigma)_{\infty}$.

Note that if $g$ is as in (6.2), then $g(\eta)=\widetilde{\sigma} a(\eta) \widetilde{\sigma}_{0}+\widetilde{r}(\eta)$ for any choice of cut-off functions $\widetilde{\sigma}, \widetilde{\sigma}_{0} \in \mathcal{C}^{\infty}\left(\left[0,1[)\right.\right.$ with a resulting $\widetilde{r} \in C_{G}^{-\infty}(\Sigma)_{\infty}$. Moreover, the (pointwise) composition of such operator-families yields a map

$$
C_{G}^{\mu_{0}}(\Sigma)_{\infty} \times C_{G}^{\mu_{1}}(\Sigma)_{\infty} \longrightarrow C_{G}^{\mu_{0}+\mu_{1}}(\Sigma)_{\infty}
$$

6.2. Holomorphic Mellin symbols. A holomorphic Mellin symbol of order $\mu \in \mathbb{R}$ is a function $h: \overline{\mathbb{R}}_{+} \times \mathbb{C} \rightarrow L_{c l}^{\mu}(\partial \mathbb{B} ; \Sigma)$ depending smoothly on $t \in \overline{\mathbb{R}}_{+}$and holomorphically on $z \in \mathbb{C}$. It has its values in the Fréchet space of parameter-dependent pseudodifferential operators on the boundary of $\mathbb{B}$. Moreover we require that

$$
c_{l}(\delta):=\sup _{t \geq 0}[t]^{l}\left\|\partial_{t}^{l} h(t, \delta+i \tau)\right\| \|
$$

is a locally bounded function of $\delta \in \mathbb{R}$ for any $l \in \mathbb{N}_{0}$ and any semi-norm $\|\cdot\|$ of $L_{c l}^{\mu}\left(\partial \mathbb{B} ; \mathbb{R}_{\tau} \times \Sigma\right)$. We denote the space of all such symbols by $M_{O}^{\mu}\left(\overline{\mathbb{R}}_{+} \times \partial \mathbb{B} ; \Sigma\right)$ and write $M_{O}^{\mu}(\partial \mathbb{B} ; \Sigma)$ for the subspace of $t$-independent symbols.

With $h \in M_{O}^{\mu}\left(\overline{\mathbb{R}}_{+} \times \partial \mathbb{B} ; \Sigma\right)$ we associate an operator-family $\mathcal{S}^{\infty}\left(\partial \mathbb{B}^{\wedge}\right) \rightarrow \mathcal{S}^{\infty}\left(\partial \mathbb{B}^{\wedge}\right)$ by

$$
\left(\operatorname{op}_{M}(h)(\eta) u\right)(t, x)=\int_{\Gamma} t^{-z} h(t, z, t \eta)(\mathcal{M} u)(z, x) d z, \quad u \in \mathcal{S}^{\infty}\left(\partial \mathbb{B}^{\wedge}\right)
$$

where $\Gamma$ is an arbitrary vertical line in the complex plane (the arbitrariness is due to the holomorphy of $\mathcal{M u}$ and Cauchy's integral formula). Note that on the right-hand side of (6.4) we do not use the symbol $h(t, z, \eta)$ itself, but the 'degenerate' one $h(t, z, t \eta)$. We refer to operators of that kind as parameter-dependent Mellin pseudodifferential operators or, shortly, Mellin operators.

REMARK 6.4. If $A$ is a cone differential operator as in (2.1) then, for any $\varphi \in \mathcal{C}^{\infty}([0,1[)$,

$$
\varphi\left(\eta^{\mu}-A\right)=\varphi t^{-\mu} \operatorname{op}_{M}(h)(\eta), \quad h(t, z, \eta)=\eta^{\mu}-\sum_{j=0}^{\mu} a_{j}(t) z^{j}
$$

Mellin operators behave well under composition:

TheOREM 6.5. Let $h_{j} \in M_{O}^{\mu_{j}}\left(\overline{\mathbb{R}}_{+} \times \partial \mathbb{B} ; \Sigma\right)$ for $j=0,1$. Then

$$
\left(h_{0} \# h_{1}\right)(t, z, \eta)=\iint s^{i \tau} h_{0}(t, z+i \tau, \eta) h_{1}(s t, z, s \eta) \frac{d s}{s} d \tau
$$

defines an element $h_{0} \# h_{1} \in M_{O}^{\mu_{0}+\mu_{1}}\left(\overline{\mathbb{R}}_{+} \times \partial \mathbb{B} ; \Sigma\right)$, the so-called Leibniz product, and

$$
\operatorname{op}_{M}\left(h_{0}\right)(\eta) \operatorname{op}_{M}\left(h_{1}\right)(\eta)=\operatorname{op}_{M}\left(h_{0} \# h_{1}\right)(\eta) \quad \forall \eta \in \Sigma .
$$

The right-hand side of $(6.5)$ is understood as an oscillatory integral in a suitable sense. 
6.3. The calculus of flat cone operators. The operator-families we now consider are, roughly speaking, those which are usual parameter-dependent pseudodifferential operators in the interior of $\mathbb{B}$, and which are parameter-dependent Mellin operators near the boundary. The global smoothing elements are flat Green symbols. Let us make this precise:

Definition 6.6. Let $\mu \in \mathbb{R}$. Then $C_{O}^{\mu}(\Sigma)$ denotes the space of all operator-families $\mathcal{C}^{\infty, \infty}(\mathbb{B}) \rightarrow$ $\mathcal{C}^{\infty, \infty}(\mathbb{B})$ of the form

$$
c(\eta)=\sigma t^{-\mu} \mathrm{op}_{M}(h)(\eta) \sigma_{0}+(1-\sigma) p(\eta)\left(1-\sigma_{1}\right)+g(\eta),
$$

where $\sigma, \sigma_{0}, \sigma_{1}$ are cut-off functions satisfying $\sigma \sigma_{0}=\sigma, \sigma \sigma_{1}=\sigma_{1}$, and

a) $h(t, z, \eta) \in M_{O}^{\mu}\left(\overline{\mathbb{R}}_{+} \times \partial \mathbb{B} ; \Sigma\right)$ is a holomorphic Mellin symbol, cf. Section 6.2 ,

b) $p(\eta) \in L_{c l}^{\mu}(2 \mathbb{B} ; \Sigma)$ is a parameter-dependent pseudodifferential operator on $2 \mathbb{B}$,

c) $g(\eta) \in C_{G}^{\mu}(\Sigma)_{\infty}$ is a Green symbol, cf. Definition 6.2.

For any choice of $0<\varrho<1$ one can achieve that the symbols $h$ and $p$ in the representation (6.6) are compatible in the sense that

$$
\varphi\left\{t^{-\mu} \mathrm{op}_{M}(h)(\eta)-p(\eta)\right\} \psi \in C_{G}^{-\infty}(\Sigma)_{\infty} \quad \forall \varphi, \psi \in \mathcal{C}_{\mathrm{comp}}^{\infty}(] \varrho, 1[) .
$$

In order to formulate the calculus in a smooth way, we shall fix such a $\varrho$ and shall always assume this compatibility relation to be satisfied. Moreover, we assume the involved cut-off functions $\sigma, \sigma_{0}, \sigma_{1}$ to be identically 1 in a neighborhood of $[0, \varrho]$. Occasionally, we shall write $c(\eta)=\mathrm{op}(h, p, g)$ if $c(\eta)$ is as in (6.6).

THEOREM 6.7. The pointwise composition of operator-families yields a map

$$
C_{O}^{\mu_{0}}(\Sigma) \times C_{O}^{\mu_{1}}(\Sigma) \longrightarrow C_{O}^{\mu_{0}+\mu_{1}}(\Sigma) .
$$

More precisely, if $c_{j}(\eta)=\mathrm{op}\left(h_{j}, p_{j}, g_{j}\right)$ for $j=0,1$, then

$$
c_{0}(\eta) c_{1}(\eta)=\operatorname{op}\left(\left(T^{\mu_{1}} h_{0}\right) \# h_{1}, p_{0} p_{1}, \widetilde{g}\right)
$$

with a resulting Green symbol $\widetilde{g} \in C_{G}^{\mu_{0}+\mu_{1}}(\Sigma)_{\infty}$. Recall that $\left(T^{\delta} h\right)(t, z, \eta)=h(t, z+\delta, \eta)$.

The operator-families from $C_{O}^{\mu}(\Sigma)$ introduced above are a subclass of parameter-dependent pseudodifferential operators on the interior of $\mathbb{B}$. In particular, we can associate with them the usual homogeneous principal symbol

$$
\sigma_{\psi}^{\mu}(c)(y, \varrho, \eta) \in \mathcal{C}^{\infty}\left(\left(T^{*} \operatorname{int} \mathbb{B} \times \Sigma\right) \backslash 0\right)
$$

with $(y, \varrho)$ referring to variables of the cotangent bundle of int $\mathbb{B}$. In the coordinates $y=(t, x)$ near the boundary with corresponding covariables $\varrho=(\tau, \xi)$, the principal symbol has the form

$$
\sigma_{\psi}^{\mu}(c)(t, x, \tau, \xi, \eta)=t^{-\mu} a_{(\mu)}(t, x, t \tau, \xi, t \eta)
$$

with a function $a_{(\mu)}(t, x, \tau, \xi, \eta)$, which is smooth in $(t, x) \in \overline{\mathbb{R}}_{+} \times \mathbb{R}^{n}$ and $0 \neq(\tau, \xi, \eta) \in \mathbb{R}^{n+1} \times \Sigma$, and is positive homogeneous of order $\mu$ in $(\tau, \xi, \eta)$. Passing to the symbol $a_{(\mu)}(0, x, \tau, \xi, \eta)$ globally leads to the definition of the rescaled principal symbol

$$
\widetilde{\sigma}_{\psi}^{\mu}(c)(x, \tau, \xi, \eta) \in \mathcal{C}^{\infty}\left(\left(T^{*} \partial \mathbb{B} \times \mathbb{R} \times \Sigma\right) \backslash 0\right) .
$$

Roughly speaking, this rescaled symbol describes the behavior of the principal symbol in the conical singularity itself. We say that $c$ is $\mathbb{B}$-elliptic if

(E) both the principal symbol $\sigma_{\psi}^{\mu}(c)$ and the rescaled symbol $\widetilde{\sigma}_{\psi}^{\mu}(c)$ are pointwise everywhere invertible.

This condition allows the construction of a rough parametrix: 
ThEOREM 6.8. Let $c(\eta)=\operatorname{op}\left(h_{0}, p_{0}, g_{0}\right) \in C_{O}^{\mu}(\Sigma)$ be $\mathbb{B}$-elliptic. Then there exists an operator-family $b(\eta)=\operatorname{op}\left(h_{1}, p_{1}, g_{1}\right) \in C_{O}^{-\mu}(\Sigma)$ such that

$$
\begin{aligned}
& b(\eta) c(\eta)=1+\omega(t[\eta]) \operatorname{op}_{M}\left(f_{L}\right)(\eta) \omega_{0}(t[\eta])+g_{L}(\eta) \\
& c(\eta) b(\eta)=1+\omega(t[\eta]) \operatorname{op}_{M}\left(f_{R}\right)(\eta) \omega_{0}(t[\eta])+g_{R}(\eta)
\end{aligned}
$$

with an arbitrary choice of cut-off functions $\omega, \omega_{0}$, Mellin symbols $f_{L}, f_{R} \in M_{O}^{-\infty}\left(\overline{\mathbb{R}}_{+} \times \partial \mathbb{B} ; \Sigma\right)$, and flat Green symbols $g_{L}, g_{R} \in C_{G}^{0}(\Sigma)_{\infty}$. Moreover, $f_{L}=\left(T^{\mu} h_{1}\right) \# h_{0}-1$ and $f_{R}=\left(T^{-\mu} h_{0}\right) \# h_{1}-1$ on $[0,1[$.

Hence, $\mathbb{B}$-elliptic symbols can be inverted up to smoothing remainders. However, this parametrix is not quite satisfactory, since a smoothing Mellin term is present and the Green symbols still have order 0 . To improve the quality of the remainder, one has to enlarge the calculus substantially (and has to pose additional ellipticity conditions). The elements of this enlarged calculus will be described in the next sections.

6.4. Green symbols with asymptotics. Let $E^{0}, E^{1}$ be Banach spaces and $\kappa^{j}=\left\{\kappa_{\varrho}^{j} \mid \varrho>\right.$ $0\} \subset \mathcal{L}\left(E^{j}\right)$ a strongly continuous group on $E^{j}$, i.e. $\kappa_{1}^{j}=1$ and $\kappa_{\varrho}^{j} \kappa_{\sigma}^{j}=\kappa_{\varrho \sigma}^{j}$. We refer to $\kappa^{j}$ as the group action of $E^{j}$.

A function $a$ in $\mathcal{C}^{\infty}\left(\Sigma, \mathcal{L}\left(E^{0}, E^{1}\right)\right)$ is said to be a symbol of order $\mu \in \mathbb{R}$, if

$$
\left\|\kappa_{1 /\langle\eta\rangle}^{1} \partial_{\eta}^{\alpha} a(\eta) \kappa_{\langle\eta\rangle}^{0}\right\|_{\mathcal{L}\left(E^{0}, E^{1}\right)} \leq c_{\alpha}\langle\eta\rangle^{\mu-|\alpha|}
$$

uniformly in $\eta \in \Sigma$ and for all multi-indices $\alpha$. We write $a \in S^{\mu}\left(\Sigma ; E^{0}, E^{1}\right)$.

A function $a \in \mathcal{C}^{\infty}\left(\Sigma \backslash\{0\}, \mathcal{L}\left(E^{0}, E^{1}\right)\right)$ is called (twisted) homogeneous of order $\mu \in \mathbb{R}$, if

$$
a(\varrho \eta)=\varrho^{\mu} \kappa_{\varrho}^{1} a(\eta) \kappa_{1 / \varrho}^{0} \quad \forall \varrho>0, \eta \neq 0 .
$$

We shall denote the space of such functions by $S^{(-\mu)}\left(\Sigma ; E^{0}, E^{1}\right)$. The standard concept of classical (polyhomogeneous) symbols having asymptotic expansions into homogeneous components extends to this operator-valued situation, resulting in the space $S_{c l}^{\mu}\left(\Sigma ; E^{0}, E^{1}\right)$.

As a straightforward modification, one can admit $E^{1}$ to be a Fréchet space, which is the projective limit of Banach spaces, $E^{1}=\lim _{k \in \mathbb{N}} E_{k}^{1}$ with $E_{1}^{1} \hookleftarrow E_{2}^{1} \hookleftarrow \ldots$, such that the group action on $E_{1}^{1}$ induces (by restriction) the group actions on all $E_{k}^{1}, k \in \mathbb{N}$. Then we simply set

$$
S_{c l}^{\mu}\left(\Sigma ; E^{0}, E^{1}\right)=\bigcap_{k \in \mathbb{N}} S_{c l}^{\mu}\left(\Sigma ; E^{0}, E_{k}^{1}\right) .
$$

In the sequel we shall introduce various distribution spaces on $\partial \mathbb{B}^{\wedge}=\mathbb{R}_{+} \times \partial \mathbb{B}$. The group action $\kappa$ always will be that induced by

$$
\left(\kappa_{\varrho} u\right)(t, x)=\varrho^{\frac{n+1}{2}} u(\varrho t, x), \quad u \in \mathcal{C}_{\mathrm{comp}}^{\infty}\left(\partial \mathbb{B}^{\wedge}\right) .
$$

Definition 6.9. Let $\gamma, \theta \in \mathbb{R}$ and $\theta>0$. An asymptotic type $Q \in \operatorname{As}(\gamma, \theta)$ is a finite set of triples $(q, l, L)$, where $q$ is a complex number with $\frac{n+1}{2}-\gamma-\theta<\operatorname{Re} q<\frac{n+1}{2}-\gamma, l \in \mathbb{N}_{0}$, and $L \subset \mathcal{C}^{\infty}(\partial \mathbb{B})$ is a finite-dimensional space of smooth functions. We shall write $Q=O$ if $Q$ is the empty set.

The conjugate type $\bar{Q} \in \operatorname{As}(\gamma, \theta)$ to $Q$ is the set of triples $(\bar{q}, l, L)$, where $(q, l, L) \in Q$.

With an asymptotic type $Q=\left\{\left(q_{j}, l_{j}, L_{j}\right) \mid j=0, \ldots, N\right\} \in \operatorname{As}(\gamma, \theta)$ we associate a finitedimensional subspace of smooth functions supported in $[0,1[\times \partial \mathbb{B}$, namely

$$
\mathcal{E}_{Q}=\left\{(t, x) \mapsto \omega(t) \sum_{j=0}^{N} \sum_{k=0}^{l_{j}} u_{j k}(x) t^{-q_{j}} \log ^{k} t \mid u_{j k} \in L_{j}\right\} .
$$

Here $\omega$ is an arbitrary cut-off function. 
Definition 6.10. Let $s \in \mathbb{R}$ and $Q \in \operatorname{As}(\gamma, \theta)$ be an asymptotic type. Then define

$$
\begin{aligned}
\mathcal{K}_{p, Q}^{s, \gamma}\left(\partial \mathbb{B}^{\wedge}\right) & =\mathcal{E}_{Q} \oplus \underset{\varepsilon>0}{\lim _{\varepsilon>0}} \mathcal{K}_{p}^{s, \gamma+\theta-\varepsilon}\left(\partial \mathbb{B}^{\wedge}\right) \\
\mathcal{S}_{Q}^{\gamma}\left(\partial \mathbb{B}^{\wedge}\right) & =\left\{u \in \mathcal{K}_{2, Q}^{\infty, \gamma}\left(\partial \mathbb{B}^{\wedge}\right) \mid(1-\omega) u \in \mathcal{S}\left(\partial \mathbb{B}^{\wedge}\right)\right\} .
\end{aligned}
$$

In case $Q$ is the empty set, we shall write $\mathcal{K}_{p, \theta}^{s, \gamma}\left(\partial \mathbb{B}^{\wedge}\right)$ and $\mathcal{S}_{\theta}^{\gamma}\left(\partial \mathbb{B}^{\wedge}\right)$, respectively.

The spaces are clearly independent of the choice of the cut-off function. Moreover, they are Fréchet and can be written as projective limits of Banach spaces. This allows us to introduce Green operators as operator-valued symbols in the above sense.

Definition 6.11. Let $Q \in \operatorname{As}(-\gamma, \theta), Q^{\prime} \in \operatorname{As}\left(\gamma^{\prime}, \theta^{\prime}\right)$ be given asymptotic types. We denote by $R_{G}^{\mu}\left(\Sigma ; \gamma, \theta, Q ; \gamma^{\prime}, \theta^{\prime}, Q^{\prime}\right)$ the space of all functions $a: \Sigma \rightarrow \mathcal{L}\left(\mathcal{K}_{2}^{0, \gamma}\left(\partial \mathbb{B}^{\wedge}\right), \mathcal{K}_{2}^{0, \gamma^{\prime}}\left(\partial \mathbb{B}^{\wedge}\right)\right)$ with

$$
a \in \bigcap_{s \in \mathbb{R}} S_{c l}^{\mu}\left(\Sigma ; \mathcal{K}_{2}^{s, \gamma}\left(\partial \mathbb{B}^{\wedge}\right), \mathcal{S}_{Q^{\prime}}^{\gamma^{\prime}}\left(\partial \mathbb{B}^{\wedge}\right)\right), \quad a^{*} \in \bigcap_{s \in \mathbb{R}} S_{c l}^{\mu}\left(\Sigma ; \mathcal{K}_{2}^{s,-\gamma^{\prime}}\left(\partial \mathbb{B}^{\wedge}\right), \mathcal{S}_{Q}^{-\gamma}\left(\partial \mathbb{B}^{\wedge}\right)\right) .
$$

Here, the * refers to the pointwise adjoint with respect to the scalar product of $\mathcal{K}_{2}^{0,0}\left(\partial \mathbb{B}^{\wedge}\right)$. Moreover, we set

$$
R_{G}^{\mu}\left(\Sigma ; \gamma, \theta ; \gamma^{\prime}, \theta^{\prime}\right)=\bigcup_{Q, Q^{\prime}} R_{G}^{\mu}\left(\Sigma ; \gamma, \theta, Q ; \gamma^{\prime}, \theta^{\prime}, Q^{\prime}\right)
$$

and write $R_{G}^{\mu}\left(\Sigma ; \gamma, \gamma^{\prime}, \theta\right)$ if $\theta=\theta^{\prime}$.

As an example, the flat Green symbols in Definition 6.2 are symbols of that type, namely

$$
R_{G}^{\mu}(\Sigma)_{\infty}=\bigcap_{\gamma, \gamma^{\prime}, \theta, \theta^{\prime}} R_{G}^{\mu}\left(\Sigma ; \gamma, \theta, O ; \gamma^{\prime}, \theta^{\prime}, O\right) \text {. }
$$

It is often important to know that Green symbols have integral kernels with a specific structure. Set

$$
\mathcal{S}_{0}^{\gamma}\left(\partial \mathbb{B}^{\wedge}\right)=\left\{u \in \mathcal{K}_{2}^{\infty, \gamma}\left(\partial \mathbb{B}^{\wedge}\right) \mid(1-\omega) u \in \mathcal{S}\left(\partial \mathbb{B}^{\wedge}\right),\left(\log ^{k} t\right) \omega u \in \mathcal{K}_{2}^{\infty, \gamma}\left(\partial \mathbb{B}^{\wedge}\right) \forall k \in \mathbb{N}_{0}\right\}
$$

ThEOREM 6.12. Let $a: \Sigma \rightarrow \mathcal{L}\left(\mathcal{K}_{2}^{0, \gamma}\left(\partial \mathbb{B}^{\wedge}\right), \mathcal{K}_{2}^{0, \gamma^{\prime}}\left(\partial \mathbb{B}^{\wedge}\right)\right)$ for given asymptotic types $Q \in \operatorname{As}(-\gamma, \theta)$ and $Q^{\prime} \in \operatorname{As}\left(\gamma^{\prime}, \theta^{\prime}\right)$. Then $a \in R_{G}^{\mu}\left(\Sigma ; \gamma, \theta, Q ; \gamma^{\prime}, \theta^{\prime}, Q^{\prime}\right)$ if and only if a satisfies 6.1] with a kernel

$$
k_{a} \in S_{c l}^{\mu}(\Sigma) \widehat{\otimes}_{\pi} \mathcal{S}_{Q^{\prime}}^{\gamma^{\prime}}\left(\partial \mathbb{B}^{\wedge}\right) \widehat{\otimes}_{\Gamma} \mathcal{S}_{\bar{Q}}^{-\gamma}\left(\partial \mathbb{B}^{\wedge}\right),
$$

where we have set

$$
\mathcal{S}_{Q^{\prime}}^{\gamma^{\prime}}\left(\partial \mathbb{B}^{\wedge}\right) \widehat{\otimes}_{\Gamma} \mathcal{S}_{\bar{Q}}^{-\gamma}\left(\partial \mathbb{B}^{\wedge}\right)=\left[\mathcal{S}_{Q^{\prime}}^{\gamma^{\prime}}\left(\partial \mathbb{B}^{\wedge}\right) \widehat{\otimes}_{\pi} \mathcal{S}_{0}^{-\gamma}\left(\partial \mathbb{B}^{\wedge}\right)\right] \cap\left[\mathcal{S}_{0}^{\gamma^{\prime}}\left(\partial \mathbb{B}^{\wedge}\right) \widehat{\otimes}_{\pi} \mathcal{S}_{\bar{Q}}^{-\gamma}\left(\partial \mathbb{B}^{\wedge}\right)\right] .
$$

To define general Green symbols on $\mathbb{B}$ we need to introduce some function spaces on $\mathbb{B}$ :

$$
\mathcal{C}^{\infty, \gamma}(\mathbb{B})=\left\{u \in \mathcal{C}^{\infty}(\operatorname{int} \mathbb{B}) \mid \omega u \in \mathcal{S}_{0}^{\gamma}\left(\partial \mathbb{B}^{\wedge}\right)\right\}, \quad \mathcal{C}_{Q}^{\infty, \gamma}(\mathbb{B})=\left\{u \in \mathcal{C}^{\infty}(\text { int } \mathbb{B}) \mid \omega u \in \mathcal{S}_{Q}^{\gamma}\left(\partial \mathbb{B}^{\wedge}\right)\right\}
$$

These are subspaces of $\mathcal{H}_{p}^{s, \gamma}(\mathbb{B})$, independent of the choice of the involved cut-off function $\omega$. We shall write $\mathcal{C}_{\theta}^{\infty, \gamma}(\mathbb{B})$ if $Q=O \in \operatorname{As}(\gamma, \theta)$ is the empty asymptotic type.

Now we define $C_{G}^{-\infty}\left(\Sigma ; \gamma, \theta, Q ; \gamma^{\prime}, \theta^{\prime}, Q^{\prime}\right)$ as the space of all functions $r: \Sigma \rightarrow \mathcal{L}\left(\mathcal{H}_{2}^{0, \gamma}(\mathbb{B}), \mathcal{H}_{2}^{0, \gamma^{\prime}}(\mathbb{B})\right)$ such that

$$
r \in \bigcap_{s \in \mathbb{R}} S^{-\infty}\left(\Sigma ; \mathcal{H}_{2}^{s, \gamma}(\mathbb{B}), \mathcal{C}_{Q^{\prime}}^{\infty, \gamma^{\prime}}(\mathbb{B})\right), \quad r^{*} \in \bigcap_{s \in \mathbb{R}} S^{-\infty}\left(\Sigma ; \mathcal{H}_{2}^{s,-\gamma^{\prime}}(\mathbb{B}), \mathcal{C}_{Q}^{\infty,-\gamma}(\mathbb{B})\right),
$$

where $*$ refers to the adjoint with respect to the scalar-product of $\mathcal{H}_{2}^{0,0}(\mathbb{B})$ and all spaces are equipped with the trivial group action $\kappa \equiv 1$.

Similar as in Definition 6.1 above, such operator-families possess an integral kernel

$$
k_{r} \in \mathcal{S}(\Sigma) \widehat{\otimes}_{\pi} \mathcal{C}_{Q^{\prime}}^{\infty, \gamma^{\prime}}(\mathbb{B}) \widehat{\otimes}_{\Gamma} \mathcal{C}_{\bar{Q}}^{\infty,-\gamma}(\mathbb{B}),
$$

where

$$
\mathcal{C}_{Q^{\prime}}^{\infty, \gamma^{\prime}}(\mathbb{B}) \widehat{\otimes}_{\Gamma} \mathcal{C}_{\bar{Q}}^{\infty,-\gamma}(\mathbb{B})=\left[\mathcal{C}_{Q^{\prime}}^{\infty, \gamma^{\prime}}(\mathbb{B}) \widehat{\otimes}_{\pi} \mathcal{C}^{\infty,-\gamma}(\mathbb{B})\right] \cap\left[\mathcal{C}^{\infty, \gamma^{\prime}}(\mathbb{B}) \widehat{\otimes}_{\pi} \mathcal{C}_{\bar{Q}}^{\infty,-\gamma}(\mathbb{B})\right]
$$


Taking the union over all possible asymptotic types leads to the spaces $C_{G}^{-\infty}\left(\Sigma ; \gamma, \theta ; \gamma^{\prime}, \theta^{\prime}\right)$ and $C_{G}^{-\infty}\left(\Sigma ; \gamma, \gamma^{\prime}, \theta\right)$ if $\theta=\theta^{\prime}$.

Definition 6.13. Let $C_{G}^{\mu}\left(\Sigma ; \gamma, \theta ; \gamma^{\prime}, \theta^{\prime}\right)$ denote the space of all operator-families $g(\eta): \mathcal{C}^{\infty, \gamma}(\mathbb{B}) \rightarrow$ $\mathcal{C}^{\infty, \gamma^{\prime}}(\mathbb{B}), \eta \in \Sigma$, such that

$$
g(\eta)=\sigma a(\eta) \sigma_{0}+r(\eta)
$$

for cut-off functions $\sigma, \sigma_{0}$, and $a \in R_{G}^{\mu}\left(\Sigma ; \gamma, \theta ; \gamma^{\prime}, \theta^{\prime}\right), r \in C_{G}^{-\infty}\left(\Sigma ; \gamma, \theta ; \gamma^{\prime}, \theta^{\prime}\right)$.

The pointwise composition of such operator-families yields a map

$$
C_{G}^{\mu_{0}}\left(\Sigma ; \gamma^{\prime}, \theta_{1}^{\prime} ; \gamma^{\prime \prime}, \theta^{\prime \prime}\right) \times C_{G}^{\mu_{1}}\left(\Sigma ; \gamma, \theta ; \gamma^{\prime}, \theta^{\prime}\right) \longrightarrow C_{G}^{\mu_{0}+\mu_{1}}\left(\Sigma ; \gamma, \theta ; \gamma^{\prime \prime}, \theta^{\prime \prime}\right)
$$

Let us finish this subsection with a result we shall need for the proof of our main theorem.

LEMmA 6.14. Let $\gamma \in \mathbb{R}$ and $0<\varepsilon<\frac{1}{2}$. Then

$$
C_{G}^{0}(\Sigma ; \gamma, 1, O ; \gamma, 1, O) \subset C_{G}^{0}(\Sigma ; \gamma, 1-2 \varepsilon, O ; \gamma+2 \varepsilon, 1-2 \varepsilon, O)
$$

Proof. Let $g(\eta) \in C_{G}^{0}(\Sigma ; \gamma, 1, O ; \gamma, 1, O)$. By Definition 6.13, we can write $g(\eta)=\sigma a(\eta) \sigma_{0}+$ $r(\eta)$ with $a(\eta) \in R_{G}^{0}(\Sigma ; \gamma, 1, O ; \gamma, 1, O)$ and $r(\eta) \in C_{G}^{-\infty}(\Sigma ; \gamma, 1, O ; \gamma, 1, O)$. We now have to show that

$$
a(\eta) \in R_{G}^{0}(\Sigma ; \gamma, 1-2 \varepsilon, O ; \gamma+2 \varepsilon, 1-2 \varepsilon, O)
$$

and that $r(\eta) \in C_{G}^{-\infty}(\Sigma ; \gamma, 1-2 \varepsilon, O ; \gamma+2 \varepsilon, 1-2 \varepsilon, O)$. We restrict ourselves to the proof of (6.12), since the symbol $r(\eta)$ can be treated in an analogous, even simpler way. By Theorem 6.12 it suffices to show that

$$
\mathcal{S}_{1}^{\gamma}\left(\partial \mathbb{B}^{\wedge}\right) \widehat{\otimes}_{\Gamma} \mathcal{S}_{1}^{-\gamma}\left(\partial \mathbb{B}^{\wedge}\right) \subset \mathcal{S}_{1-2 \varepsilon}^{\gamma+2 \varepsilon}\left(\partial \mathbb{B}^{\wedge}\right) \widehat{\otimes}_{\Gamma} \mathcal{S}_{1-2 \varepsilon}^{-\gamma}\left(\partial \mathbb{B}^{\wedge}\right)
$$

(recall that we write $\mathcal{S}_{\theta}^{\gamma}=\mathcal{S}_{O}^{\gamma}$ if $O \in \operatorname{As}(\gamma, \theta)$ is the empty asymptotic type). Clearly,

$$
\mathcal{S}_{1}^{\gamma}\left(\partial \mathbb{B}^{\wedge}\right) \widehat{\otimes}_{\Gamma} \mathcal{S}_{1}^{-\gamma}\left(\partial \mathbb{B}^{\wedge}\right) \subset \mathcal{S}_{1}^{\gamma}\left(\partial \mathbb{B}^{\wedge}\right) \widehat{\otimes}_{\pi} \mathcal{S}_{0}^{-\gamma}\left(\partial \mathbb{B}^{\wedge}\right)=\mathcal{S}_{1-2 \varepsilon}^{\gamma+2 \varepsilon}\left(\partial \mathbb{B}^{\wedge}\right) \widehat{\otimes}_{\pi} \mathcal{S}_{0}^{-\gamma}\left(\partial \mathbb{B}^{\wedge}\right)
$$

where the last identity follows directly from the definition of the involved spaces. By Proposition 4.5 of 27 (in the version for operators on $\partial \mathbb{B}^{\wedge}$ ) we have

$$
\mathcal{S}_{1}^{\gamma}\left(\partial \mathbb{B}^{\wedge}\right) \widehat{\otimes}_{\Gamma} \mathcal{S}_{1}^{-\gamma}\left(\partial \mathbb{B}^{\wedge}\right)=\bigcap_{0 \leq \sigma \leq 1} \mathcal{S}_{\sigma}^{\gamma}\left(\partial \mathbb{B}^{\wedge}\right) \widehat{\otimes}_{\pi} \mathcal{S}_{1-\sigma}^{-\gamma}\left(\partial \mathbb{B}^{\wedge}\right)
$$

Inserting $\sigma=2 \varepsilon+\delta, \delta>0$ small, yields

$$
\mathcal{S}_{1}^{\gamma}\left(\partial \mathbb{B}^{\wedge}\right) \widehat{\otimes}_{\Gamma} \mathcal{S}_{1}^{-\gamma}\left(\partial \mathbb{B}^{\wedge}\right) \subset \mathcal{S}_{2 \varepsilon+\delta}^{\gamma}\left(\partial \mathbb{B}^{\wedge}\right) \widehat{\otimes}_{\pi} \mathcal{S}_{1-2 \varepsilon-\delta}^{-\gamma}\left(\partial \mathbb{B}^{\wedge}\right) \subset \mathcal{S}_{0}^{\gamma+2 \varepsilon}\left(\partial \mathbb{B}^{\wedge}\right) \widehat{\otimes}_{\pi} \mathcal{S}_{1-2 \varepsilon-\delta}^{-\gamma}\left(\partial \mathbb{B}^{\wedge}\right) .
$$

Passing to the intersection over all $\delta>0$ gives

$$
\mathcal{S}_{1}^{\gamma}\left(\partial \mathbb{B}^{\wedge}\right) \widehat{\otimes}_{\Gamma} \mathcal{S}_{1}^{-\gamma}\left(\partial \mathbb{B}^{\wedge}\right) \subset \mathcal{S}_{0}^{\gamma+2 \varepsilon}\left(\partial \mathbb{B}^{\wedge}\right) \widehat{\otimes}_{\pi} \mathcal{S}_{1-2 \varepsilon}^{-\gamma}\left(\partial \mathbb{B}^{\wedge}\right)
$$

This finishes the proof.

6.5. Meromorphic Mellin symbols. An asymptotic type for Mellin symbols $P$ is a set of triples $(p, n, N)$ with $p \in \mathbb{C}, n \in \mathbb{N}_{0}$, and $N$ a finite-dimensional subspace of finite rank operators from $L^{-\infty}(\partial \mathbb{B})$. Moreover, we require that $\pi_{\mathbb{C}} P \cap\{z \in \mathbb{C} \mid-\delta \leq \operatorname{Re} z \leq \delta\}$ is a finite set for each $\delta>0$, where

$$
\pi_{\mathbb{C}} P=\{p \in \mathbb{C} \mid(p, n, N) \in P \text { for some } n, N\} .
$$

We shall write $P=O$ if $P$ is the empty set.

A meromorphic Mellin symbol with asymptotic type $P$ is a meromorphic function $f: \mathbb{C} \rightarrow$ $L^{-\infty}(\partial \mathbb{B})$ with poles at most in the points of $\pi_{\mathbb{C}} P$. Moreover it satisfies: If $(p, n, N) \in P$, then the principal part of the Laurent series of $f$ in $p$ is of the form $\sum_{k=0}^{n} R_{k}(z-p)^{-k-1}$ with $R_{k} \in N$; if $\chi \in \mathcal{C}^{\infty}(\mathbb{C})$ is a $\pi_{\mathbb{C}} P$-excision function (i.e. identically zero in an $\varepsilon$-neighborhood around $\pi_{\mathbb{C}} P$ 
and identically 1 outside the $2 \varepsilon$-neighborhood), then $c(\delta)=\|\|(\chi f)(\delta+i \tau)\|\|$ is a locally bounded function in $\delta \in \mathbb{R}$ for each semi-norm of $L^{-\infty}\left(\partial \mathbb{B} ; \mathbb{R}_{\tau}\right)=\mathcal{S}\left(\mathbb{R}_{\tau}, L^{-\infty}(\partial \mathbb{B})\right)$.

As in (6.4) we can associate with meromorphic Mellin symbols a pseudodifferential operator. Now, however, the operator will depend on the choice of the line. Letting $\Gamma_{1 / 2-\delta}$ denote the vertical line $\{\operatorname{Re} z=1 / 2-\delta\}$ we define $\operatorname{op}_{M}^{\delta}(f)$ by

$$
\left(\operatorname{op}_{M}^{\delta}(f) u\right)(t, x)=\int_{\Gamma_{\frac{1}{2}-\delta}} t^{-z} f(t, z)(\mathcal{M} u)(z, x) d z
$$

Of course, we have to require that none of the poles of $f$ lies on the chosen line; this we shall always assume implicitly.

Definition 6.15. Let $\gamma, \mu \in \mathbb{R}$ and $k \in \mathbb{N}$. Then $C_{M+G}^{\mu}(\Sigma ; \gamma, \gamma-\mu, k)$ denotes the space of all operator-families $\mathcal{C}^{\infty, \gamma}(\mathbb{B}) \rightarrow \mathcal{C}^{\infty, \gamma-\mu}(\mathbb{B})$ of the form

$$
\omega(t[\eta])\left(\sum_{j=0}^{k-1} \sum_{|\alpha|=0}^{j} t^{-\mu+j} \operatorname{op}_{M}^{\gamma_{j \alpha}-\frac{n}{2}}\left(f_{j \alpha}\right) \eta^{\alpha}\right) \widetilde{\omega}(t[\eta])+g(\eta)
$$

where $\omega, \widetilde{\omega}$ are arbitrary cut-off functions, $g \in C_{G}^{\mu}(\Sigma ; \gamma, \gamma-\mu, k)$, the $f_{j \alpha}$ are meromorphic Mellin symbols with certain asymptotic types $P_{j \alpha}$, and $\gamma_{j \alpha} \in \mathbb{R}$ with $\gamma-j \leq \gamma_{j \alpha} \leq \gamma$.

Changing the cut-off functions in (6.14) only yields remainders in $C_{G}^{\mu}(\Sigma ; \gamma, \gamma-\mu, k)$.

6.6. The calculus of cone pseudodifferential operators. For $\gamma, \mu \in \mathbb{R}$ and $k \in \mathbb{N}$ let

$$
C^{\mu}(\Sigma ; \gamma, \gamma-\mu, k)=C_{O}^{\mu}(\Sigma)+C_{M+G}^{\mu}(\Sigma ; \gamma, \gamma-\mu, k)
$$

with $C_{O}^{\mu}(\Sigma)$ from Definition 6.6 and $C_{M+G}^{\mu}(\Sigma ; \gamma, \gamma-\mu, k)$ as in Definition 6.15. The elements of that space are operator-families $\mathcal{C}^{\infty, \gamma}(\mathbb{B}) \rightarrow \mathcal{C}^{\infty, \gamma-\mu}(\mathbb{B})$. Pointwise composition induces a map

$$
C^{\mu_{0}}\left(\Sigma ; \gamma-\mu_{1}, \gamma-\mu_{1}-\mu_{0}, k\right) \times C^{\mu_{1}}\left(\Sigma ; \gamma, \gamma-\mu_{1}, k\right) \longrightarrow C^{\mu_{0}+\mu_{1}}\left(\Sigma ; \gamma, \gamma-\mu_{1}-\mu_{0}, k\right)
$$

REMARK 6.16. Let $c(\eta) \in C^{\mu}(\Sigma ; \gamma, \gamma-\mu, k)$. Then, for each fixed $\eta, c(\eta)$ induces continuous maps

$$
c(\eta): \mathcal{H}_{p}^{s, \gamma}(\mathbb{B}) \longrightarrow \mathcal{H}_{p}^{s-\mu, \gamma-\mu}(\mathbb{B})
$$

for any $s \in \mathbb{R}$ and $1<p<\infty$. Moreover, to any asymptotic type $Q \in \operatorname{As}(\gamma, k)$ there exists a type $Q^{\prime} \in \operatorname{As}(\gamma-\mu, k)$ such that

$$
c(\eta): \mathcal{C}_{Q}^{\infty, \gamma}(\mathbb{B}) \longrightarrow \mathcal{C}_{Q^{\prime}}^{\infty, \gamma-\mu}(\mathbb{B})
$$

The construction of the parameter-dependent parametrix in Section 3.2 implicitly relies on ideas from the edge calculus; in particular, in (3.8) the notion of principal edge symbol is used to obtain a description of $\left(\eta^{\mu}-\underline{\widehat{A}}\right)^{-1}$. We shall therefore recall those symbolic structures. In order to put this into perspective, we shall give - although this will not be needed in this paper - two theorems that show how the different types of ellipticity imply the existence of parametrices of corresponding quality.

Let $c(\eta) \in C^{\mu}(\Sigma ; \gamma, \gamma-\mu, k)$ be given; then

$$
c(\eta)=\sigma t^{-\mu} \operatorname{op}_{M}^{\gamma-\frac{n}{2}}(h)(\eta) \sigma_{0}+(1-\sigma) p(\eta)\left(1-\sigma_{1}\right)+(m+g)(\eta),
$$

where the first two terms are as in (6.6) and $(m+g)(\eta)$ is as in (6.14). Since $(m+g)(\eta)$ has, in particular, a smooth distributional kernel, $c(\eta)$ is a parameter-dependent pseudodifferential operator on the interior of $\mathbb{B}$, and we can associate with it the principal symbol and rescaled symbol as in (6.7) and (6.8), respectively.

The principal edge symbol is

$$
\sigma_{\wedge}^{\mu}(c)(\eta)=t^{-\mu} \operatorname{op}_{M}^{\gamma-\frac{n}{2}}\left(h_{0}\right)(\eta)+\omega(t|\eta|)\left(\sum_{j=0}^{k-1} \sum_{|\alpha|=j} t^{-\mu+j} \operatorname{op}_{M}^{\gamma_{j \alpha}-\frac{n}{2}}\left(f_{j \alpha}\right) \eta^{\alpha}\right) \widetilde{\omega}(t|\eta|)+g_{(\mu)}(\eta)
$$


where $h_{0}(z, \eta)=h(0, z, \eta)$ and $g_{(\mu)}(\eta)$ is the homogeneous principal symbol of $g(\eta) \in R_{G}^{\mu}(\Sigma ; \gamma, \gamma-$ $\mu, k)$. We consider the principal edge symbol as an operator-family

$$
\sigma_{\wedge}^{\mu}(c)(\eta): \mathcal{K}_{p}^{s, \gamma}\left(\partial \mathbb{B}^{\wedge}\right) \longrightarrow \mathcal{K}_{p}^{s-\mu, \gamma-\mu}\left(\partial \mathbb{B}^{\wedge}\right), \quad \eta \neq 0,
$$

for $s \in \mathbb{R}$ and $1<p<\infty$.

Finally, the conormal symbol of $c(\eta)$ is the meromorphic function with values in $L_{c l}^{\mu}(\partial \mathbb{B})$ given by

$$
\sigma_{M}^{\mu}(c)(z)=h(0, z, 0)+f_{00}(z): H_{p}^{s}(\partial \mathbb{B}) \longrightarrow H_{p}^{s-\mu}(\partial \mathbb{B}), \quad z \in \mathbb{C} .
$$

We shall call $c(\eta) \in C^{\mu}(\Sigma ; \gamma, \gamma-\mu, k)$ elliptic, if

(E) both $\sigma_{\psi}^{\mu}(c)$ and $\widetilde{\sigma}_{\psi}^{\mu}(c)$ are pointwise everywhere invertible (i.e. $c(\eta)$ is $\mathbb{B}$-elliptic),

$\left(\mathrm{E}_{\wedge}\right)$ the principal edge symbol $\sigma_{\wedge}^{\mu}(c)$ in $(6.17)$ is pointwise everywhere invertible.

Here, the second condition initially is required to hold for some $s$ and $p$; but then it holds for all. As soon as $c(\eta)$ satisfies one of the conditions $(\mathrm{E})$ or $\left(\mathrm{E}_{\wedge}\right)$, the conormal symbol will be meromorphically invertible. It is bijective on the vertical line $\Gamma_{\frac{n+1}{2}-\gamma}$ in case $c(\eta)$ satisfies $\left(\mathrm{E}_{\wedge}\right)$.

TheOREm 6.17. Assume $c(\eta) \in C^{\mu}(\Sigma ; \gamma, \gamma-\mu, k)$ satisfies condition (E) and the conormal symbol is invertible on the line $\Gamma_{\frac{n+1}{2}-\gamma}$. Then there exists a $b(\eta) \in C^{-\mu}(\Sigma ; \gamma-\mu, \gamma, k)$ such that

$$
b(\eta) c(\eta)-1 \in C_{G}^{0}(\Sigma ; \gamma, \gamma, k), \quad c(\eta) b(\eta)-1 \in C_{G}^{0}(\Sigma ; \gamma-\mu, \gamma-\mu, k) .
$$

This (still rough) parametrix $b(\eta)$ is uniquely determined modulo $C_{G}^{-\mu}(\Sigma ; \gamma-\mu, \gamma, k)$.

Theorem 6.18. Let $c(\eta) \in C^{\mu}(\Sigma ; \gamma, \gamma-\mu, k)$ be elliptic. Then there exists a $b(\eta) \in C^{-\mu}(\Sigma ; \gamma-$ $\mu, \gamma, k)$ such that

$$
b(\eta) c(\eta)-1 \in C_{G}^{-\infty}(\Sigma ; \gamma, \gamma, k), \quad c(\eta) b(\eta)-1 \in C_{G}^{-\infty}(\Sigma ; \gamma-\mu, \gamma-\mu, k) .
$$

The parametrix $b(\eta)$ is uniquely determined modulo $C_{G}^{-\infty}(\Sigma ; \gamma-\mu, \gamma, k)$.

\section{References}

[1] J. Brüning and R. Seeley. An index theorem for regular singular operators. Amer. J. Math. 110:659-714, 1988.

[2] J. Cheeger. On the spectral geometry of spaces with cone-like singularities. Proc. Nat. Acad. Sci. USA 76:21032106, 1979.

[3] S. Coriasco, E. Schrohe, J. Seiler. Bounded imaginary powers of cone differential operators. Math. Z., to appear. (Preprint math.AP/0106008).

[4] S. Coriasco, E. Schrohe, J. Seiler. Differential operators on conic manifolds: Maximal regularity and parabolic equations. Bull. Soc. Roy. Sci. Liège 70: 207-229, 2001.

[5] G. Dore, A. Venni. On the closednes of the sum of two operators. Math. Z. 196: 189-201, 1987.

[6] Yu. Egorov, B.-W. Schulze. Pseudodifferential Operators, Singularities, Applications., Birkhäuser Verlag 1997.

[7] J.B. Gil. Heat trace asymptotics for cone differential operators. PhD thesis, Potsdam 1998.

[8] J.B. Gil. Full asymptotic expansion of the heat trace for non-self-adjoint elliptic cone operators. To appear in Math. Nachr.

[9] J.B. Gil, P. Loya. On the noncommutative residue and the heat trace expansion on conic manifolds To appear in Manuscripta Math.

[10] J.B. Gil, G.A. Mendoza. Adjoints of elliptic cone operators. Preprint, Temple University, Philadelphia, 2001. To appear in Amer. J. Math.

[11] J.B. Gil, B.-W. Schulze, J. Seiler. Cone pseudodifferential operators in the edge symbolic calculus. Osaka J. Math. 37: 221-260, 2000.

[12] M. Lesch. Operators of Fuchs type, Conical Singularities, and Asymptotic Methods. Teubner-Texte Math. 136, Teubner-Verlag, 1997.

[13] P. Loya. Complex Powers of Differential Operators on Manifolds with Conical Singularities. To appear in $J$. Anal. Math.

[14] P. Loya. On the Resolvent of Differential Operators on Conic Manifolds. To appear in Comm. Anal. Geom.

[15] R. Melrose. Transformation of boundary value problems. Acta Math. 147:149-236, 1981.

[16] E. Mooers. Heat kernel asymptotics on manifolds with conic singularities. J. Anal. Math. 78: 1-36, 1999.

[17] B. Plamenevskij. Algebras of pseudodifferential operators. Nauka, Moscow 1986 (in Russian). 
[18] E. Schrohe. Spaces of weighted symbols and weighted Sobolev spaces on manifolds. In H.O. Cordes, B. Gramsch, H. Widom (eds.), Pseudodifferential operators, Oberwolfach (1986), pp. 360-377. LN Math. 1256, Springer Verlag, 1987.

[19] E. Schrohe, B.W. Schulze. Edge-degenerate boundary value problems on cones. In Evolution equations and their applications in physical and life sciences, Bad Herrenalb (1998), pp. 159-173. Marcel Dekker, 2001.

[20] E. Schrohe, J. Seiler. Ellipticity and invertibility in the cone algebra on $L_{p}$-Sobolev spaces. Int. Eq. Oper. Th. 41: 93-114, 2001.

[21] B.-W. Schulze. The Mellin pseudo-differential calculus on manifolds with corners. In H. Triebel et al. (eds.), Symposium 'Analysis on Manifolds with Singularities', Breitenbrunn 1990, Teubner-Texte Math. 131, TeubnerVerlag, 1992.

[22] B.-W. Schulze. Pseudo-differential Operators on Manifolds with Singularities. North-Holland, 1991.

[23] R. Seeley. Complex powers of an elliptic operator. In Amer. Math. Soc. Proc. Symp. Pure Math., volume 10: 288-307, 1967.

[24] R. Seeley. The resolvent of an elliptic boundary problem. Amer. J. Math. 91: 889-920, 1969.

[25] R. Seeley. Norms and domains of the complex powers Az. Amer. J. Math. 93: 299-309, 1971.

[26] J. Seiler. Pseudodifferential Calculus on Manifolds with Non-compact Edges. PhD-thesis, Institut für Mathematik, Potsdam, 1997.

[27] J. Seiler. The cone algebra and a kernel characterization of Green operators. In J.B. Gil et al. (eds.), Approaches to Singular Analysis, pp. 1-29. Birkhäuser Verlag, 2001.

Universität Potsdam, Institut für Mathematik, Postfach 6015 53, 14415 Potsdam, Germany

E-mail address: schrohe@math.uni-potsdam.de

Universität Potsdam, Institut für Mathematik, Postfach 6015 53, 14415 Potsdam, Germany

E-mail address: seiler@math.uni-potsdam.de 